OPEN ACCESS

Edited by:

George E. Barreto,

Pontifical Xavierian University,

Colombia

Reviewed by:

Hari S. Sharma,

Uppsala University, Sweden Wolfgang Härtig,

Leipzig University, Germany Ignacio Torres-Aleman,

Consejo Superior de Investigaciones Cientificas (CSIC), Spain

Christiane Charriaut-Marlangue, Institut National de la Santé et de la Recherche Médicale (INSERM)

France

${ }^{*}$ Correspondence: Alla B. Salmina allasalmina@mail.ru

Received: 23 March 2018 Accepted: 17 July 2018

Published: 06 August 2018

Citation:

Osipova ED, Komleva YK, Morgun AV, Lopatina OL, Panina YA, Olovyannikova RY, Vais EF, Salmin W and Salmina $A B$ (2018) Designing in vitro Blood-Brain Barrier Models Reproducing Alterations in Brain Aging.

Front. Aging Neurosci. 10:234. doi: 10.3389/fnagi.2018.00234

\section{Designing in vitro Blood-Brain Barrier Models Reproducing Alterations in Brain Aging}

\author{
Elena D. Osipova ${ }^{1,2}$, Yulia K. Komleva ${ }^{1,2}$, Andrey V. Morgun ${ }^{3}$, Olga L. Lopatina ${ }^{1,2}$, \\ Yulia A. Panina ${ }^{1}$, Raissa Ya. Olovyannikova ${ }^{1}$, Elizaveta F. Vais ${ }^{1}$, Vladimir V. Salmin ${ }^{3}$ \\ and Alla B. Salmina ${ }^{1,2 *}$
}

\begin{abstract}
'Department of Biochemistry, Medical, Pharmaceutical \& Toxicological Chemistry, Krasnoyarsk State Medical University named after Prof. V.F. Voino-Yasenetsky, Krasnoyarsk, Russia, ${ }^{2}$ Research Institute of Molecular Medicine \& Pathobiochemistry, Krasnoyarsk State Medical University named after Prof. V.F. Voino-Yasenetsky, Krasnoyarsk, Russia, ${ }^{3}$ Department of Medical and Biological Physics, Krasnoyarsk State Medical University named after Prof. V.F.

Voino-Yasenetsky, Krasnoyarsk, Russia
\end{abstract}

Blood-brain barrier (BBB) modeling in vitro is a huge area of research covering study of intercellular communications and development of BBB, establishment of specific properties that provide controlled permeability of the barrier. Current approaches in designing new BBB models include development of new (bio) scaffolds supporting barriergenesis/angiogenesis and BBB integrity; use of methods enabling modulation of BBB permeability; application of modern analytical techniques for screening the transfer of metabolites, bio-macromolecules, selected drug candidates and drug delivery systems; establishment of 3D models; application of microfluidic technologies; reconstruction of microphysiological systems with the barrier constituents. Acceptance of idea that BBB in vitro models should resemble real functional activity of the barrier in different periods of ontogenesis and in different (patho) physiological conditions leads to proposal that establishment of BBB in vitro model with alterations specific for aging brain is one of current challenges in neurosciences and bioengineering. Vascular dysfunction in the aging brain often associates with leaky BBB, alterations in perivascular microenvironment, neuroinflammation, perturbed neuronal and astroglial activity within the neurovascular unit, impairments in neurogenic niches where microvascular scaffold plays a key regulatory role. The review article is focused on aging-related alterations in BBB and current approaches to development of "aging" BBB models in vitro.

Keywords: blood-brain barrier, neurodegeneration, aging, model in vitro, cell senescence

\section{INTRODUCTION}

Brain aging is one of the most intriguing issues in modern neurobiology, physiology and cell biology. Effects of aging on pivotal brain functions, i.e., cognition and social behavior, are well-known, however, the molecular basis on neurological deficits appearing in the aging brain and showing strong tendency to further redoubling is not fully understood. Numerous experimental and clinical data suggest that the complex of alterations is of great importance for the development of functionally incompetent brain in aged individuals. Such complex includes excessive neuronal death associated with impaired neurogenesis and gray matter shrinking, gliopathy associated with neuroinflammation, excitotoxicity and demyelination, disturbed cerebral microcirculation, 
imbalanced production of regulatory molecules, altered bloodbrain barrier (BBB) permeability and impaired functional brain networks (Peters, 2006; Desai et al., 2010; Antonenko and Flöel, 2014; Bajaj et al., 2017). In this context, age-associated brain disorders might be considered as a particular case of accelerated aging or as an example of disturbed aging process. Also, it is clear that several exogenous factors may affect brain aging either negatively (stress, intoxication etc.) or positively (physical exercise, cognitive training, balanced diet etc.). Most importantly, deciphering molecular mechanisms of their action would help in preventing brain dysfunction or in developing upto-date therapeutic strategy aimed to control aging process in the brain.

$\mathrm{BBB}$ is a highly specialized complex of cells within so-called neurovascular unit (NVU) that are responsible for brain tissue protection, controlled bidirectional transport of fluids, endogenous and exogenous (macro) molecules, immune and progenitor cells trafficking, primarily, at the level of cerebral capillaries. In mammals, BBB/NVU includes cerebral endothelial cells lying on the thin basement membrane (BM), pericytes (PC), perivascular astroglia, neurons and microglia (Abbott and Friedman, 2012; Banks, 2016). A main component of the $\mathrm{BBB}$ is a monolayer of brain microvessel endothelial cells (BMECs) that are characterized by high mitochondrial content, low fenestration level, substantial expression of tight junction proteins, small perivascular space, pronounced coverage with astroglial end-feet, and expression of wide spectrum of transporters and receptors (Figure 1). Intercellular communications play prominent role in the regulation of $\mathrm{BBB}$ establishment in embryos and in the early neonatal period (barriergenesis), maintenance of BBB structural and functional integrity in the adult brain, in acquisition of barrier properties in newly-formed cerebral vessels in (patho) physiological conditions (reparative angiogenesis, plasticityassociated angiogenesis; Salmina et al., 2015b; Dudvarski Stankovic et al., 2016; Lecuyer et al., 2016; Osipova et al., 2018).

Current interest for molecular mechanisms of $\mathrm{BBB}$ development and function is based on real clinical needs. Particularly, overcoming the barrier for the targeted delivery of drugs to the brain tissue, monitoring the barrier status and controlling its permeability in pathological conditions (neuroinflammation, stroke, brain edema), testing new drugscandidates in in vitro and in silico BBB models are among top technologies expected to transform the therapy of central nervous system disorders (Albrecht et al., 2016; Zhang Y. Y. et al., 2016; Semyachkina-Glushkovskaya et al., 2017a,b; Bolwerk et al., 2018).

BBB dysfunction in the aging brain relates to various mechanisms, including loss of structural barrier integrity, lower functional coupling of cells contributing to the barrier establishment, PC loss, disturbed activity of BMECs within clonogenic niches (i.e., neurogenic niche, oligovasculogenic niche), altered activity of $\mathrm{BBB}$ molecular transporters, and impaired activity of glymphatic system (Zeevi et al., 2010). Thus, simplest characteristic of aging BBB is a loss of structural and functional integrity resulting in pathological permeability

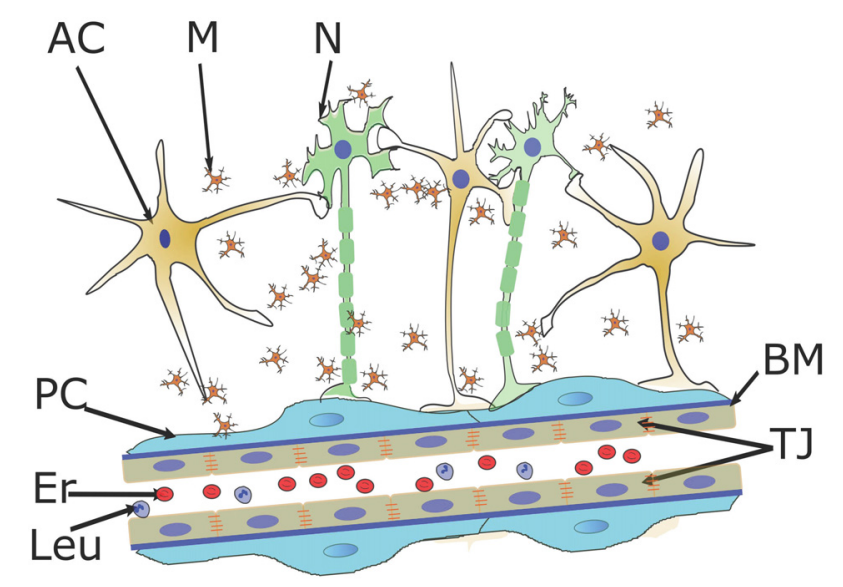

FIGURE 1 | Blood-brain barrier (BBB) structure and intercellular communications within the neurovascular unit (NVU). Brain microvessel endothelial cells (BMECs), pericytes (PC), basement membrane (BM), perivascular astroglial cells (ACs), microglia (M) and neurons (N) form the NVU where endothelial cell layer controls bidirectional transport of macromolecules and fluids, trafficking of immune and progenitor cells to the brain tissue. NVU serves as a platform for neuron-astroglia metabolic coupling, gliovascular control of microcirculation, glia- and pericyte-mediated control of BBB permeability and angiogenesis, vascular support of neurogenesis. Er, erythrocytes; Leu, leukocytes; TJ, tight junctions.

and development of abnormal barrier-related events (edema formation, neuroinflammation, insufficient clearance of brain metabolites). Paradoxically, even BBB breakdown associated with enhanced $\mathrm{BBB}$ permeability is a key property of aged brain, targeted drug delivery to the brain tissue remains an unresolved problem in gerontology (Erdö et al., 2017). Thus, reconstruction of "aging" phenotype of $\mathrm{BBB}$ in vitro would be very useful in a huge area of experimental and clinical applications.

\section{BRIEF OVERVIEW OF BBB MODELS IN VITRO: CURRENT OPPORTUNITIES AND CHALLENGES}

$\mathrm{BBB}$ modeling in vitro is a rapidly developing area of research which covers study of intercellular communications and development of $\mathrm{BBB}$, establishment of barrier properties pivotal for BBB controlled permeability, development of new scaffolds for the growth of BMECs and application of modern analytical techniques for screening barrier permeability for selected drug candidates and drug delivery systems. In the majority of cases, the following factors are critical for obtaining the fully competent BBB/NVU models in vitro. First of all, nature of cellular constituents of the model (mature cells isolated from brain issue, or undifferentiated cells of embryonic origin further subjected to proliferation and desired differentiation in vitro or induced pluripotent stem cells (iPSCs)-derived cells) is of great importance. Cells of different origin may have very specific properties in relation to the characteristics of real BBB (i.e., level of fenestration of endothelial cells, perivascular morphology of astroglial cells (ACs), or degree of cell differentiation). 
Second, establishment of monolayer or multilayer analog of the barrier should be taken into the consideration according to the general task of modeling. As an example, in vitro monolayer endothelial barrier might be sufficient for some screening procedures in experimental neuropharmacology but is almost useless for studying barriergenesis or complex intercellular communications within the NVU. Third, still there is no appropriate artificial analog of the BM underlying endothelial cells layer in cerebral capillaries, therefore, production and application of (bio) scaffolds or permeable membranes optimal for endothelial cells functional activity are highly recommended. Fourth, stability of the model and its structural and functional integrity should be achieved and easily maintained in vitro. Such barrier properties provide reliable experimental data and guarantee reproducibility of results obtained. Fifth, recent progress in microfabrication technologies allows designing microfluidic models and microphysiological systems that resemble many of the crucial properties of real $\mathrm{BBB}$ in the context of blood flow-mediated effects on endothelial cell layer, and finally, a model should allow some desired manipulations with the cells or microenvironment in order to reconstruct conditions typical for different phases of brain development or brain pathologies. As an example, one may manipulate with the expression profile of $\mathrm{BBB} / \mathrm{NVU}$ cells, may include non-NVU cells or stem cell-derived cells into the model, or expose the model to the action of exogenous physical, chemical or biological factors (Lippmann et al., 2011; Khilazheva et al., 2015; Ruck et al., 2015; Kuvacheva et al., 2016).

In sum, technology of $\mathrm{BBB}$ modeling in vitro consists of the following steps: (1) selection of appropriate cells (primary cultures, cell lines, stem/progenitor cells-derived cells) known as components of NVU/BBB (i.e., endothelial cells, PC, astrocytes etc.) with the special focus on the origin and properties of endothelial cells (i.e., BMECs, human umbilical vein endothelial cells (HUVECs) etc.); (2) reconstruction of NVU/BBB microenvironment by seeding the cells in the appropriate medium and in the designed microarchitecture (i.e., 2D model, 3D model, spheroid model, or models with contacting or non-contacting cells in a transwell) on scaffolds supporting their growth and interactions (i.e., gelatin, polylactic acid, biopolymers etc.) in static or microfluidic conditions; (3) model validation by the assessment of barrier's structural and functional integrity and selective transport activity: measurements of transendothelial electric resistance (TEER) which reflects the integrity of endothelial layer and paracellular permeability, analysis of expression of tight junction and adhesion proteins (i.e., zonula occludens 1 (ZO1), junctional adhesion molecule (JAM), occludins, claudins (CLDNs), vascular endothelial cadherins (VE-cadherins) etc.), influx and efflux transporters like glucose transporters (GLUT), monocarboxylate transporters (MCT), P-glycoprotein (Pgp), receptors for advanced glycation end products (RAGE), transferrin receptors; and evaluation of the barrier's permeability for various molecules and complexes (i.e., liposomes, dextrans, dyes, labeled ligands etc.); and (4) application of the model to the given research tasks (i.e., assessment of drug transport or cells trafficking, analysis of intercellular communications or angiogenesis-related events).

Currently, there are various $\mathrm{BBB}$ models in vitro that can be classified according to their general properties: (1) monocellular/monolayer models (consisting of BMECs) vs. multicellular/co-culture models (including BMECs, PC, ACs etc.); (2) static models (with stationary extracellular fluid) vs. microfluidic models (with controlled flow of fluid mimicking natural blood flow in cerebral microvessels); (3) brain cell-derived models (i.e., based on BMECs and astrocytes) vs. non brain cell-derived models (i.e., based on HUVEC or other cells lines) according to the origin of cells included into the model; and (4) models obtained from primary cultures vs. those obtained from the established cell lines. Also, $\mathrm{BBB}$ models in vitro might be restricted to the exact stage of ontogenesis (i.e., BBB model derived from antenatal or neonatal brain cells to study early events in barriergenesis) or to some pathological conditions (i.e., BBB model specific for neurodegeneration or neuroinflammation; Garberg et al., 2005; Helms et al., 2016; Bosworth et al., 2018; Figure 2).

It is obvious that advantages and disadvantages of either BBB in vitro model arise from their principal characteristics. For instance, widely-used transwell models allow testing intercellular communications but properties of scaffolds have a special significance, whereas spheroid cultures require no scaffolds but they do not reconstruct natural architecture of the barrier (Ruck et al., 2015), microfluidic models are assumed to be optimal for studying drug pharmacokinetics and allow desired reconfiguration of fluid flow within the device but they are rather expensive and possess high technical requirements (Bonakdar et al., 2017).

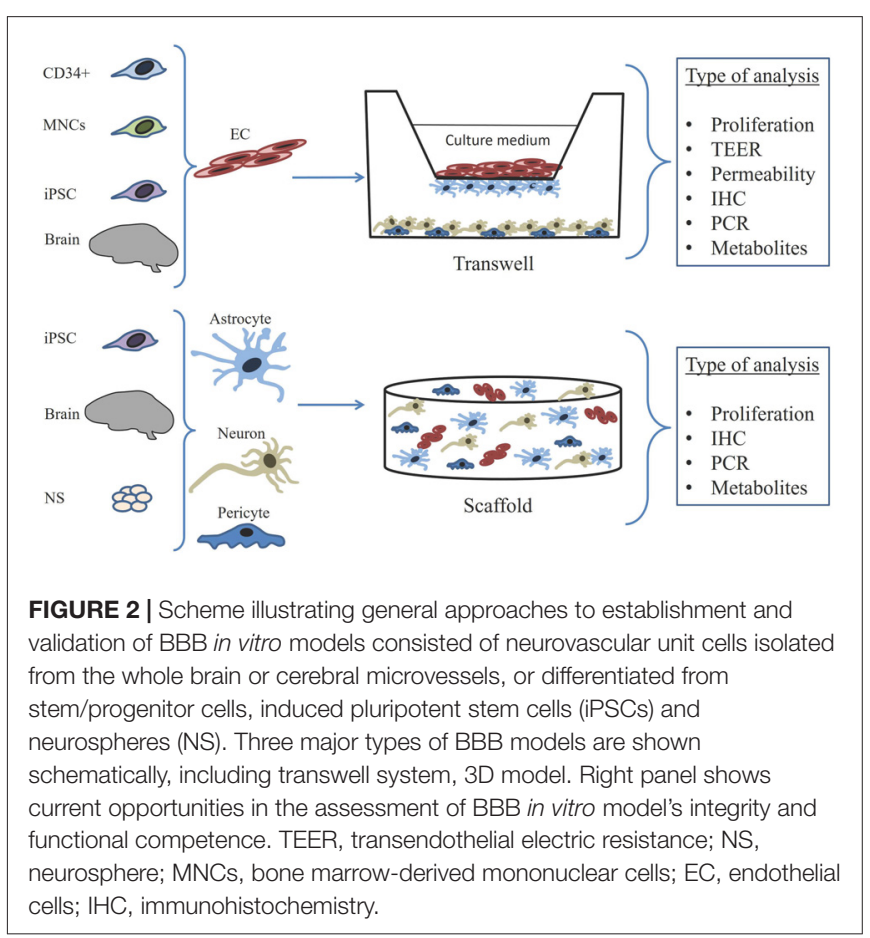


Diversity of $\mathrm{BBB}$ models allows performing pre-clinical screening of drug candidate and studying barriergenesis, transport machinery, and pathological conditions associated with the dysfunction of the NVU (Stanimirovic et al., 2015). Even "ideal" BBB models in vitro do not exist yet, however, several attempts to produce renewable, controlled, scalable and fully functional BBB models have been realized. As an example, BBB models based on cells originated from embryonic or adult stem cells became to be rather popular. In such protocol, BMECs or astrocytes are differentiated from immature stem cells, then they are phenotyped to get the populations with characteristics of desired cell type, and are further co-cultured under appropriate conditions (Malinovskaya et al., 2016). Very recent achievements in modeling BBB in vitro have been connected with the application of human iPSCsbased protocols for getting BMECs, PC and other perivascular cells representing high degree of similarity to natural $\mathrm{BBB}$ (Bosworth et al., 2018; Ribecco-Lutkiewicz et al., 2018). One can expect that further progress in the establishment of $\mathrm{BBB}$ models in vitro might be done with the application of gene-targeted strategies to modify expression of proteins directly involved into the regulation of $\mathrm{BBB}$ integrity. For example, Cre-Lox protocol has been successfully used for the disruption of Smad proteins acting downstream of TGF- $\beta$ receptors in cerebral endothelial cells (Li et al., 2011) or for the inactivation of $\beta$-catenins involved in the stabilization of endothelial tight junctions (Liebner et al., 2008). Therefore, these protocols might be further applied for creating novel BBB models with target modification of barrier properties in vitro. Analogous prospects arise from the application of precise gene editing technologies, i.e., clustered regularly interspaced short palindromic repeats (CRISPR)/Cas9-based protocol enabling manipulation with functional properties of $\mathrm{BBB} / \mathrm{NVU}$ cells (Zhou et al., 2018), or optogenetic approaches either in vivo and in vitro.

Development of microphysiological systems containing $\mathrm{BBB}$ in vitro model opens new stage in the establishment of functionally competent $\mathrm{BBB}$ in vitro. Combination of microfluidic technology and establishment of multi-tissue ensembles (i.e., neurons, liver cells, placenta cells etc.) allows studying complex inter-tissue communications relevant for various (patho) physiological conditions, performing drug screening, and creating novel test-systems for the development of drug delivery protocols (Brown et al., 2015; Phan et al., 2017; Edington et al., 2018).

\section{PROPERTIES OF BBB/NVU CONSTITUENTS IN AGING BRAIN}

Phenomenon of brain aging is in the focus of neurobiologists and neurologists. Aging is a risk factor for specific types of neurodegeneration, i.e., in Alzheimer's disease (Guerreiro and Bras, 2015), however, pathological brain aging itself represents aggravated or completely altered program of natural senescence caused by exogenous and endogenous factors. It is interesting, that regardless type of aging, neuroplasticity reserve seems to be preserved in various brain areas (Cotelli et al., 2012), even it is still debated whether pathological neurodegeneration is a type of excessive aging process (Ghosh et al., 2011). Moreover, some other factors could affect brain physiological and pathological aging. It was found that volumes of subcortical structures were much more preserved in physiological aging women comparing to men (Kiraly et al., 2016), thus suggesting that-at least in humans-male brains might be more susceptible to aging. However, some contradictory data exist as well (Greenberg et al., 2008). In pathological aging, i.e., in Alzheimer's disease development, female brains seem to be more susceptible to acquiring senescent phenotype (Zhao et al., 2016), presumably, due to specific features of metabolism or because of alterations in signaling pathways coupled to receptors of growth factors and neurosteroids.

Anyway, chronic neurodegeneration in aging brain is always accompanied by the phenomenon of vascular aging (Donato et al., 2015). It is a complex gradual process resulting in NVU dysfunction, impairment of neurogenesis and angiogenesis. As an example, in retina, progressive vascular damage in aging rats is a multistep process consisting of thickening of the basal capillary membrane, disorganization of PC cytoskeleton at the earliest stage followed by microvessel remodeling and angiogenesis at the latter stage (Hughes et al., 2006). Endothelial cells, PC, and perivascular astroglia are implicated in the pathogenesis of cerebral vascular aging. Interestingly enough, brain vascular aging and endothelial leakage in humans may start in hippocampal microvessels, thereby preceding hippocampal atrophy and cognitive decline (Montagne et al., 2015). It should be also noted that hippocampus demonstrates the highest levels of amyloid-induced hyper vascularization and leaky BBB in Alzheimer's type of neurodegeneration (Desai et al., 2009; Biron et al., 2011), suggesting that accelerated cerebral vascular aging and neurodegeneration-mediated angiopathy could have causative relationship with the activity of adult neurogenic niches responsible for hippocampus-supported learning and memory: active neurogenic events correspond to the sites of elevated BBB permeability (Lin et al., 2015; Pozhilenkova et al., 2017). As a result of endothelial, PC and astroglial impairments, brain aging is always associated with reduced cerebral capillary blood flow and altered gliovascular local control of microcirculation (Desjardins et al., 2014), BBB breakdown (Elahy et al., 2015), and disturbed synaptic plasticity evident as long-term potentiation (LTP) deficits in brain regions with pathological $\mathrm{BBB}$ permeability (Blau et al., 2012).

Endothelial dysfunction is a hallmark of normal aging. Senescent endothelial cells are characterized by higher susceptibility to oxidative stress, reduced proliferation and sensitivity to the action of pro-angiogenic factors, excessive cell death (apoptosis and autophagy), compromised ability to prevent blood coagulation events, limited availability and impaired response to vasodilating factors and propensity to support chronic inflammatory process.

BMECs have some important characteristics that make them dramatically susceptible to the above-mentioned processes, 


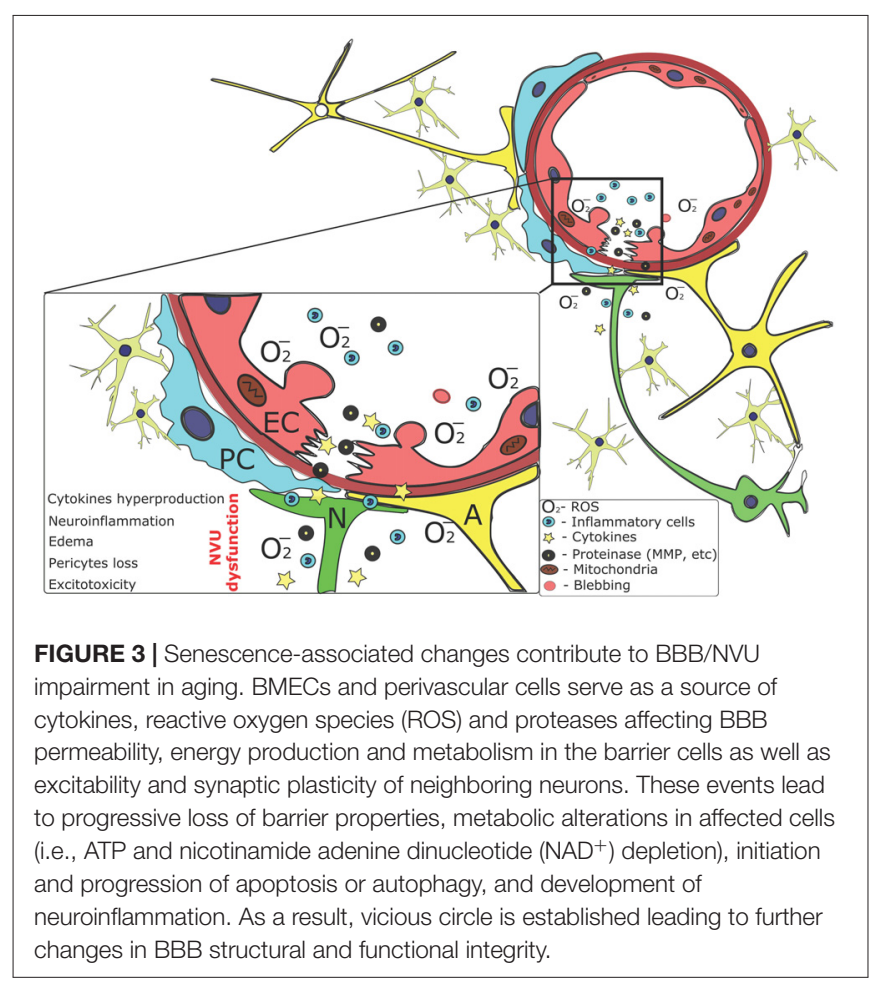

particularly, they possess high number of mitochondria (Kluge et al., 2013) and demonstrate strong intercellular coupling provided by tight junctions, adherence junctions and connexin channels (De Bock et al., 2011; Luissint et al., 2012; Dejana and Orsenigo, 2013). As an example, oxidative stress is a well-known marker of aging, whereas oxidative stress-induced thiol oxidation, phosphorylation, nitration and carbonylation of tight junction proteins result in BBB breakdown (Rao, 2008; Enciu et al., 2013). These mechanisms have been shown in D-galactose-induced mouse brain aging (Lei et al., 2013) and were implicated in obesity-promoted cerebrovascular aging in mice (Tucsek et al., 2014). In aged mice, deficit in tight junction coupling is accompanied by leaky BBB and neuroinflammatory events; the latter is not caused by significant leukocytes recruitment into the brain tissue (Elahy et al., 2015).

Senescent cells are usually characterized by overproduction of cytokines and proteases, excessive DNA damage response, mitochondrial dysfunction and reactive oxygen species (ROS) generation. All these mechanisms are tightly coupled and potentiate each other. As an example, excessive production of ROS supports development of neuroinflammatory response (El Assar et al., 2013) culminating in the establishment of so-called senescence-associated secretory phenotype (SASP) evident in endothelial and perivascular cells (Chen et al., 2002). Endothelial oxidative stress is associated with prominent activation of poly (ADP-ribose) polymerase needed for DNA repair, thereby resulting in nicotinamide adenine dinucleotide $+\left(\mathrm{NAD}^{+}\right)$depletion and endothelial cell death (Pacher et al., 2002). Additionally, in the aged brain, altered processing of amyloid precursor protein in endothelial cells leads to amyloid beta $(\mathrm{A} \beta)$ accumulation and its deposition in cerebral microvessels (Muche et al., 2017). Progressive accumulation of $\mathrm{A} \beta$ in brain microvessels results in the development of cerebral amyloid angiopathy (CAA) and supports persistence of SASP in NVU cells. All these events are always accompanied by mitochondrial dysfunction, insufficient ATP production, accelerated mitophagy and aberrant mitochondrial biogenesis in BMECs, PC and perivascular glial cells (Caja and Enríquez, 2017; Karnewar et al., 2018). Finally, SASP-associated matrix metalloproteinase and cytokines release dramatically disrupts endothelial monolayer integrity and ultimately leads to BBB hyperpermeability, microvessel remodeling and loss of neurovascular control (Goligorsky and Hirschi, 2016; Figure 3).

In cerebral endothelium, aging-related events also include elevated VEGF signaling and lowered NO availability in senescent BMECs resulting in vessels hyperpermeability and hypervascularity (Oakley and Tharakan, 2014). Whether or not VEGF action in the aging brain corresponds to the altered expression of its alternative isoforms that may either increase or decrease BBB permeability, support or suppress angiogenesis (Woolard et al., 2009) remains to be evaluated. Besides, aging affects activity of several transporters expressed in BMECs. Particularly, glucose uptake via GLUT is reduced in the aging rat microvessels (Mooradian et al., 1991), thereby suggesting functional link between aging-associated cerebral glucose hypometabolism and $\mathrm{BBB}$ impairments. $\mathrm{A} \beta$ clearance through $\mathrm{BBB}$ was found to be altered in aging rats due to abnormal expression of low-density lipoprotein receptorrelated protein-1 (LRP-1) and Pgp in BMECs (Silverberg et al., 2010). Aberrant expression of Pgp in aging human $\mathrm{BBB}$ results in disturbed cerebral pharmacokinetics of several drugs known as Pgp substrates (van Assema et al., 2012). Thus, aging-affected BMECs are characterized by progressive loss of permeability controlling mechanisms resulting in deregulated cerebral metabolism of endogenous molecules and xenobiotics.

PC dysfunction and loss are often seen in chronic neurodegeneration and, particularly, in aging. Age-dependent vascular damage in pericyte-deficient mice preceded chronic neurodegeneration and cognitive decline (Bell et al., 2010). Expression of senescence markers in brain $\mathrm{PC}-\beta$-galactosidase activity and p16 (INK4a) - was associated with compromised BBB integrity in senescence-prone mice (Yamazaki et al., 2016). In the rat frontal cortex and hippocampus, age-related changes in PC microstructure include elevation of mitochondria number, and this phenomenon is much more prominent comparing to BMECs (Hicks et al., 1983). Thus, loss of PC coverage could be partially compensated by stimulated metabolic activity in remaining cells. Indeed, even excessive PC loss can be effectively counteracted in the brain without evident changes in BBB permeability in mice (Villaseñor et al., 2017). PC always show prominent structural plasticity and their distal processes are rather dynamic (Berthiaume et al., 2018) but whether such properties are maintained in normal aging of $\mathrm{BBB}$ remains to be clarified. In sum, aging-related changes in cerebral PC mainly caused by their excessive cell death 
and microstructural rearrangements in the remaining cells might be partially compensated at the earliest stages of $\mathrm{BBB}$ breakdown.

Astroglia is affected in aging because of oxidative stress and development of local inflammation. As a result, neuron-astrocyte metabolic coupling and astrocyte-endothelial interactions are impaired, thereby leading to aberrant neuronal activity and BBB breakdown in a region-specific manner (Rodríguez et al., 2014). Aging-associated progressive telomere shortening and development of SASP occur in astroglial and endothelial cells (Bitto et al., 2010; Chinta et al., 2013; Morgan et al., 2018), thus contributing to local production of pro-inflammatory cytokines. Besides that, astrocytes in the aging mouse brain are very sensitive to the stimulatory action of activated microglia (Clarke et al., 2018), thereby contributing to the establishment of pro-inflammatory microenvironment in the perivascular area. In aging mice, alterations in paravascular drainage mechanisms provided by glymphatic system were associated with abnormal AQP4 polarization, thereby suggesting aberrant astroglial activity (Kress et al., 2014). Recent transcriptomic analysis of multiple mice brain regions showed that aging astrocytes up-regulate genes encoding proteins that are required for synaptic remodeling and astroglial activation (Boisvert et al., 2018). Moreover, senescent mouse astrocytes demonstrate higher ability to contribute to synapse elimination and show clear brain regional specialization (Boisvert et al., 2018). However, it should be taken into consideration that there is a shortage of data obtained precisely in perivascular astroglia in aging brain, therefore, the question exists whether the observed agingassociated changes in astrocytes could be safely extrapolated on particular NVU ACs whose end-feet are tightly connected with BMECs.

Astrocytes are well-known producers of lactate in active brain regions, and this metabolite could be used by neuronal cells to support their extremely high energetic needs, or could be transported and/or utilized by BMECs to control their mitochondrial activity and angiogenesis-related events (Salmina et al., 2015b). In aging, glycolytic activity of astrocytes may be dramatically compromised: aging ACs show reduction of glycolysis, stimulation of mitochondrial biogenesis and activation of mitochondrial ATP production leading to impaired neuron-astrocyte metabolic coupling and local glucose hypometabolism (Jiang and Cadenas, 2014). These events, probably, result from the establishment of local pro-inflammatory microenvironment within the NVU, since inflammation-affected cells usually activate mitochondrial biogenesis in order to compensate for mitochondrial damage (Piantadosi and Suliman, 2012). Such mechanism could be linked to mitophagy-associated activation of NLRP3 inflammasomes whose activity results in interleukins (IL-1 $\beta$, IL-18, IL33) release and inhibition of glycolysis (Tschopp, 2011). Elevated levels of brain lactate is a hallmark of agingrelated neurodegeneration, particularly, at the presymptomatic stage in mice (Ross et al., 2010), so, exact molecular mechanisms of deregulated lactate production and transport in the aging brain remain to be assessed. Thus, aging results in astroglial activation and, presumably, impairment of astroglia-supported intercellular communications within the NVU with long-lasting effects on $\mathrm{BBB}$ permeability and angiogenesis.

Microglial effects on $B B B$ in aging is poorly understood. Actually, microglial cells are not considered as an obligatory component of the NVU, however, numerous studies reveal prominent role of activated microglia in controlling $\mathrm{BBB}$ permeability due to secretion of cytokines, chemokines, ROS and several metabolites (da Fonseca et al., 2014; Dudvarski Stankovic et al., 2016; Osipova et al., 2018). Microglia may directly interact with brain microvessels, particularly, with endothelial tip cells governing angiogenesis, thus suggesting the regulatory role of activated microglial cells in cerebral barriergenesis and angiogenesis. Microglia-released interleukins, TNF and ROS potentiate $\mathrm{BBB}$ breakdown and elevate paracellular permeability of the barrier, thereby resulting in edema formation and leukocytes trafficking in the affected brain regions. So, microglial activity in a close vicinity to the $\mathrm{BBB}$ results in promotion of angiogenesis (hypervascularity) and elevated permeability of newly formed microvessels. It may have a relation to the appearance of numerous microvessels with leaky BBB in Alzheimer's disease manifested by pronounced neuroinflammation in mice (Biron et al., 2011) as well as to the delayed angiogenesis and impaired microvessel permeability in post-stroke aged rats and humans (Buga et al., 2014).

Age-dependent activation of microglia is a widely-recognized phenomenon (Spittau, 2017), therefore, it is obvious that agingassociated alterations in BBB permeability could be partially caused by microglial cells. One can describe this mechanism as an example of low-grade chronic neuroinflammation with all the corresponding attributes: microglial cytotoxicity, complement activation, phagocytosis, development of cytokine storm, excessive clearance of cellular debris in the loci of excessive apoptosis, microglial $\mathrm{M} 2 \rightarrow \mathrm{M} 1$ polarization with the corresponding changes in cell metabolism (Chan-Ling et al., 2007; Cerbai et al., 2012). However, very recent data suggest that in addition to cytotoxic and pro-inflammatory phenotype, cortical microglial cells in aged mice may also acquire immunotolerogenic properties (Zöller et al., 2018). In general, priming and even uncontrolled activation of microglia correspond to the progression of brain aging whereas severity of such changes associates with neurological deficits and behavioral abnormalities (Norden and Godbout, 2013).

$B M$ contributes $a$ lot to the functional integrity of the $\mathrm{BBB}$, and its role might be compromised in aging, i.e., due to elevated release of MMP-2 and MMP-9 from activated ACs, microglia or PC. MMPs degrade BM proteins, thereby contributing to $\mathrm{BBB}$ breakdown (Lee et al., 2012), but they also important for microvessel remodeling and neoangiogenesis. Some data suggest that aging and Alzheimer's type of neurodegeneration might be associated with significant thickening of the barrier BM (Marques et al., 2013; Yamazaki and Kanekiyo, 2017), but whether it is important for BBB dysfunction seen in the aged brain is not clear yet. 


\section{MECHANISMS AND MARKERS OF CELL SENESCENCE: APPLICATIONS FOR NVU STUDY IN BBB MODELS IN VITRO}

Probably, the most reliable way to get an NVU/BBB model resembling properties of aging brain tissue might be in obtaining of cells from the aged animal followed by their growth in vitro within the multi-cellular ensemble (endothelial cells and perivascular cells) mimicking NVU microenvironment. However, this approach is difficult to execute because proliferation and establishment of appropriate and functionally competent intercellular contacts are very limited in the culture of cells isolated from the aged brain tissue (Salmin et al., 2017). But very recent findings suggest that neurogenic potential of cells in physiological aging might be well preserved (Boldrini et al., 2018). Therefore, aged brain-derived stem and progenitor cells might be considered as a potential source of NVU cells. Another approach is based on the induction of senescencespecific properties in NVU cells obtained at much earlier stages of brain development. In this case, appropriate protocols for inducing and detecting senescence phenotype in cells within the NVU/BBB models in vitro should be validated and applied.

Proinflammatory changes in the microenvironment of senescent cells. At the cellular level, senescence is characterized by cessation of cell proliferation and establishment of so-called SASP resulting in release of proinflammatory cytokines, chemokines growth factors, MMPs and other proteases, serpins, soluble or shed receptors and their ligands, membranederived microvesicles into the extracellular space in order to create microenvironment optimal for senescent cell (Coppé et al., 2010; Robbins, 2017). Recently, cellular senescence has been recognized as a cause of chronic neurodegeneration, i.e., in Alzheimer's disease, linking neuroinflammation to non-reversible cell aging and accumulation of non-repairable alterations. Moreover, some data suggest that aging is also accompanied by an increase in the number of SASP-expressing senescent cells of non-neuronal origin (i.e., astrocytes, microglia, endothelial cells, neural stem cells) in the brain (Chinta et al., 2015). Therefore, it could be proposed that senescence of non-neuronal cells in the aging brain could contribute to progressive impairment of $\mathrm{BBB}$ (aging endothelial cells), insufficient myelination (aging oligodendrocytes), reduced neurogenesis (aging neural stem cells) and development of sterile neuroinflammation (aging astroglial and microglial cells).

Besides, another function of SASP induction might be found in the promotion of cell plasticity and stemness as it was shown in the skin: keratinocytes exposed to the SASP initially elevate expression of stem cell markers and regenerative capacity in vivo followed by the cell cycle arrest (Ritschka et al., 2017). Whether or not such mechanism is operative in the brain, particularly, in neurogenic niches with prominent vascular scaffold architecture, remains to be evaluated, but several attempts to produce neuropinflammationspecific models of BBB have been undertaken. As an example, endothelial monolayers arranged within tube-like structures on a microfluidic platform demonstrate SASP-like response to tumor necrosis factor-alpha (TNF- $\alpha$ ) action in vitro (manifested by the production of 29 cytokines, chemokines and growth factors) and reproduce some properties of inflammation-induced BBB leakage (Cho et al., 2015). Therefore, monolayer BBB models could be used for studying SASP-related changes affecting barrier integrity. The same effect could be achieved by stimulating secretory activity of perivascular astroglia which leads to enhanced release of cytokines and chemokines, thereby supporting its paracrine action at BMECs (Osipova et al., 2018). Particularly, being stimulated with the mixture of IL- $1 \beta$ and $\mathrm{TNF} \alpha$, astrocytes in vitro produce upto 30 cytokines, chemokines and soluble mediators, such as complement, growth factors, adhesion molecules, serpins, etc. in a time-dependent manner (Choi et al., 2014). If BBB model is reconstituted consequently with different cell types, preliminary culture of astrocytes with IL- $1 \beta$ and TNF- $\alpha$ would produce SASP-resembling conditions in $24 \mathrm{~h}$ after treatment in non-toxic concentrations. Another protocol is based on genetic manipulation with signaling molecules upstream the pro-inflammatory cytokines secretion. Particularly, elevated expression of nuclear factor-kappa B (NF- $\kappa B)$ in PC leads to release of monocyte chemoattractant protein-1 (MCP-1) and IL-8 that are able to stimulate endothelial cell proliferation in skeletal muscle (LaBarbera et al., 2015). Thus, the same approach could be tested in BBB models in vitro. In sum, detecting changes in cell secretome specific for SASP is a good marker of cellular senescence, whereas targeting SASP is recognized as an approach to eliminate senescent cells and to prevent aging (Watanabe et al., 2017). Induction of SASP phenotype in BMECs, PC or astroglia is a tool for modeling $\mathrm{BBB}$ with the properties specific for aging brain.

Inflammasome activation and insulin resistance (IR) in senescent cells. Activation of cell senescence program is always associated with inflammasome induction, particularly, in endothelial cells of large vessels where ROS-driven expression of cryopyrin, NOD-like receptor 3 (NLRP3) inflammasomes well explains elevated release of major pro-inflammatory cytokine IL-1 $\beta$ and acquisition of senescent phenotype (Yin et al., 2017). The same is true for the development of peripheral IR characteristic for so-called inflamm-aging (Bauernfeind et al., 2016). Expression of NLRP3 inflammasomes accompanied by increased expression of MMP-2 and reduced TEER was registered in the BBB in vitro model exposed to poly(I:C; Małecki et al., 2017), but whether such approach might be useful in establishing $\mathrm{BBB}$ model specific for the aging brain remains unclear.

Brain cells are equipped with various components of insulin signaling machinery. Alzheimer's type of neurodegeneration is believed to be a particular case of IR and impaired glucose tolerance in the brain tissue (Thambisetty et al., 2013). IR results in aberrant transport and reception of insulin in the brain tissue, thereby contributing to abnormal processing of amyloid precursor protein and excessive $\mathrm{A} \beta$ accumulation, altered metabolism of glucose and secondary changes in lactatedriven mechanisms (neuron-astrocyte metabolic coupling, 
angiogenesis; Willette et al., 2015; Neth and Craft, 2017). In humans, brain aging is associated with progressive loss of insulin receptors (Frölich et al., 1998), and very recent studies suggest that vulnerability of different brain regions to $A \beta$ toxicity corresponds to their sensitivity to local insulin action (Mullins et al., 2017).

Link of cellular senescence and IR has been demonstrated in several cell types, i.e in hepatocytes (Aravinthan et al., 2015), cardiomyocytes (Boudina, 2013). Thus, taken into the consideration that aging is often accompanied by IR, and it is true for brain (Baranowska-Bik and Bik, 2017), one may assume that induction of IR in NVU cells could make them more sensitive to the action of aging-promoting factors in vitro. It should be noted that IR prevents effective repair of endothelial layer and suppresses reparative angiogenesis, thereby promoting vascular aging (Avogaro et al., 2013). Thus, disruption of insulin sensing and signaling in NVU cells would help achieving senescence-prone phenotype for study BBB breakdown in aging brain.

Depleted $\mathrm{NAD}^{+}$levels in senescent cells. Glucose intolerance in microvessel endothelial cells results in altered glucose uptake and acceleration of their senescence due to suppression of activity of $\mathrm{NAD}^{+}$-dependent histone deacetylases sirtuins (SIRTs; Mortuza et al., 2013). Interesting to note, that elevation of $\mathrm{NAD}^{+}$levels in endothelial cells by up-regulating nicotinamide phosphoribosyltransferase (Nampt) delays acquiring the senescence phenotype and promotes angiogenesis (Borradaile and Pickering, 2009). Thus, manipulating $\mathrm{NAD}^{+}$bioavailability, i.e., via $\mathrm{NAD}^{+}$-glycohydrolases $\mathrm{CD} 38$ or $\mathrm{CD} 157$, might be a way to change cell's sensitivity to the action of aging-promoting factors in vitro (Chini, 2009; Camacho-Pereira et al., 2016; Chini et al., 2018), whereas supply of nicotinamide riboside as $\mathrm{NAD}^{+}$precursor would efficiently elevate intracellular $\mathrm{NAD}^{+}$levels (Dellinger et al., 2017) and delay cellular senescence (Zhang H. et al., 2016). CD38 and CD157 serve as receptors and enzymes with $\mathrm{NAD}^{+}$-converting activity that have been initially recognized as important regulators of immune cells functional activity (Malavasi et al., 2006). Later, it was confirmed that both the enzymes are widely expressed in the brain being predominantly found in neurons, astrocytes, and microglia (Ceni et al., 2003) where they take part in the action of neurotransmitters, coordinate neuronastrocyte metabolic coupling, regulate local immune response. In particular regions of the brain (hypothalamic area and pituitary) $\mathrm{CD} 38$ controls $\mathrm{NAD}^{+}$metabolism and neurosecretory activity required for oxytocin secretion which regulates various neurobehavioral responses, i.e., interpersonal communications, parental behavior, decision making (Lopatina et al., 2011, 2012; Higashida et al., 2012; Salmina et al., 2012; Akther et al., 2013). Expression of CD38 and CD157 is controlled by pro-inflammatory cytokines, retinoic acid, cell proliferation or differentiations status, and availability of their own ligand $\mathrm{NAD}^{+}$(Malavasi et al., 2008). In endothelial cells, inhibition of CD38 preserves nitric oxide (NO) synthase activity and NO generation, whereas activation of CD38 leads to NADPH depletion, thereby contributing to endothelial dysfunction in the heart (Reyes et al., 2015). Presumably, analogous mechanisms might be active in BMECs since production of $\mathrm{NO}$ is greatly compromised in cerebral microvessels in chronic neurodegeneration (Salmina et al., 2015a). CD38 is also expressed in retinal PC being up-regulated by pro-inflammatory cytokines, whereas application of anti-CD38 antibodies resulted in PC injury ( $\mathrm{Li}$ et al., 2012). Very recent data revealed expression of CD157 in endothelial cells and it function as an angiogenesis-regulating molecule in various tissues, including brain (Wakabayashi et al., 2018). It is tempting to speculate that aberrant expression of CD38 and CD157 in BMECs and cerebral PC might affect $\mathrm{NAD}^{+}$levels and acquisition of senescence phenotype in $\mathrm{BBB}$ in vitro models.

Oxidative stress is another mechanism causing cell senescence in vitro. Partially it is explained by oxidant-induced acute depletion of intracellular $\mathrm{NAD}^{+}$pool due to excessive activation of poly(ADP-ribose)polymerase required for efficient DNA repair. Thus, it is not surprising that one of the methods to induce cell senescence in BBB models in vitro is application of oxidants like $\mathrm{H}_{2} \mathrm{O}_{2}$. However, efficacy of $\mathrm{H}_{2} \mathrm{O}_{2}$ may differ depending on cell types and previous passages of cells (particularly, endothelial cells) that affect cell cycle and their sensitivity to the action of genotoxic agents (Yamazaki et al., 2016). However, this approach might be very effective in getting phenotypic changes characteristic for aging BBB.

Glycolysis provides partial regeneration of $\mathrm{NAD}^{+}$pool in the cells, therefore glycolytic activity of NVU cells would have an impact on their aging dynamics. Metabolic profiling of senescent fibroblasts has shown elevated activity of glycolysis that can be explained by mitochondrial dysfunction in aging cells (James et al., 2015). Similar effect has been observed in senescent astrocytes where up-regulation of glycolysis corresponded to the degree of mitochondrial overactivation, ROS production, and inflammatory response of astroglia (Cohen et al., 2017). Thus, elevated glycolytic flux might be a part of cell protective mechanism to prevent $\mathrm{NAD}^{+}$depletion and to delay cell aging.

Recently, we found that activation of GPR81 lactate receptors in BMECs in vitro resulted in mitochondrial biogenesis and was accompanied by reduced expression of MCT-1 and CD147 (Khilazheva et al., 2017). Mitochondrial biogenesis is driven by DNA damage in senescent cells and results in elevated production of ROS and cell cycle arrest (Correia-Melo et al., 2016). Thus, enhanced rate of glycolysis in senescent cells could lead to massive efflux of lactate to the extracellular space where it acts at GPR81 receptors and stimulates mitochondrial biogenesis in BMECs. The latter results in excessive production of ROS, DNA damage, and induction of DNA-damage response (DDR) which is a hallmark of aging cells.

$D D R$ in senescent cells. One of experimental approaches to induce senescence of cultured cells is based on their exposure to gamma-irradiation which can induce reparable or irreparable DNA damage (James et al., 2015). This method is effective since another important feature of senescent cells is an induction of DDR which is a prerequisite for SASP and is triggered by genomic lesions. It is generally accepted that senescent cells accumulate the phosphorylated form 
of histone $\mathrm{H} 2 \mathrm{AX}(\gamma-\mathrm{H} 2 \mathrm{AX})$ which marks sites of DNA double strand breaks (DSBs) and is required for maintaining genome integrity (Turinetto and Giachino, 2015). Telomere shortening seen in senescent cells leads to the loss of telomerebound inhibitors of ATM and other kinases involved in H2AX phosphorylation, therefore, DDR is spontaneously activated (so-called replicative senescence induced by telomere attrition; d'Adda di Fagagna, 2008; Maicher et al., 2012).

In the brain, accumulation of $\gamma-\mathrm{H} 2 \mathrm{AX}$ mainly occurs in neurons and glial cells, but some cerebral endothelial cells demonstrate presence of this marker as well (Barral et al., 2014). Hippocampal astrocytes show high levels of $\gamma$-H2AX expression in Alzheimer's type of neurodegeneration (Myung et al., 2008), endothelial cells need in $\gamma$-H2AX for their proliferation in hypoxia-driven neoangiogenesis (Chavakis et al., 2009). H2 $\mathrm{AX}^{-/-}$mice demonstrate impaired endothelial cell proliferation (but not pericyte dysfunction) associated with reduced angiogenesis (Economopoulou et al., 2009). In neurons, phosphorylation of H2AX with ATM and DNA-PK occurs in physiological brain activity: glutamatergic stimulation of neurons or exposure to novel environment result in topoisomerase II-dependent DSBs and appearance of $\gamma$-H2AX witnessing immediate early genes expression (i.e., c-fos, npas4, egr1) in activated neuronal cells (Suberbielle et al., 2013; Madabhushi et al., 2015). Thus, phosphorylated H2AX might be a good marker of DSBs and/or telomere shortening but not a specific marker of cellular senescence due to its presence in functionally active non-damages cells (Bernadotte et al., 2016). However, location of $\gamma-\mathrm{H} 2 \mathrm{AX}$ in genome could be helpful in discriminating exogenous and endogenous mechanisms of its induction (i.e., ionizing radiation and activation of transcription, respectively). For example, sub-telomeres are less responsive to external DNA damage than to endogenous stress (Seo et al., 2012).

TABLE 1 | Main molecular mechanisms and markers of aging-associated alterations in neurovascular unit (NVU).

Aging-associated alterations

Pathophysiological events in NVU

Molecular markers

Reference

in NVU

Endothelial dysfunction

Oxidative stress

Apoptosis

Impairment of tight junctions, adherence junctions, connexin channels Hyperpermeability, hypervascularity

\section{Reduced proliferation}

Astroglial dysfunction

Altered metabolism and morphology

Local glucose hypometabolism

Neuroinflammation, Inflamm-aging

Pericyte dysfunction

Microglial activation and dysfunction

Neuronal dysfunction
Pericytes loss

Neuroinflammation, Inflamm-aging

Neuronal loss

Local insulin resistance and corresponding metabolic alterations

Synaptic dysfunction
ROS overproduction, NADPH oxidase activity

Specific DNA fragmentation, phosphatidylserine exposure Altered expression of $\mathrm{Cx} 43, \mathrm{Cx} 40$, CLD5, ZO1, JAM

Elevated expression of VEGF, MMP2, MMP9, decrease of TEER, increased permeability for dyes, dextrans, liposomes

Decreased expression of Ki67, PCNA

Aberrant lactate production

Decreased expression of GLUT4, IRAP, low lactate levels

SASP phenotype (expression of inflammasomes, RAGE, HMGB1, IL-1 $\beta$, IL-18, IL-33, TNF $\alpha$, other cytokines and chemokines)

Reduced number of pericytes, decreased expression of PDGFR SASP phenotype (overproduction of cytokines, chemokines, ROS), signs of $\mathrm{M} 2 \rightarrow \mathrm{M} 1$ polarization (expression of Arg1, CD206, and Ym1 vs IL-1 $\beta$, TNF- $\alpha$, IL-6, CD16/32, CD86, CD40, iNOS)

Neurogenesis impairments, decreased expression of NeuN, DCX, excessive expression of cell death (apoptosis, autophagy) markers

Altered expression and activity of insulin receptors, GLUT, IRS, PI3K, Akt, GSK3 $\beta$

Low expression of PSD95, Synaptophysin
Freeman and Keller (2012); Sohrabji et al. (2013)

Hoffmann et al. (2001)

Lei et al. (2013); Elahy et al. (2015) Biron et al. (2011); Zhang et al. (2017)

Katsimpardi et al. (2014)

Jiang and Cadenas (2014); Goodall et al. (2018) Mosconi (2013); Camandola and Mattson (2017)

Fu et al. (2014); Chinta et al. (2015)

Bell et al. (2010); Winkler et al. (2010) Norden and Godbout (2013); Solano Fonseca et al. (2016)

Sun et al. (2013)

Akintola and van Heemst (2015)

Morrison and Baxter (2012); Mostany et al. (2013) 
In sum, various experimental approaches should be considered when $\mathrm{BBB}$ model is established in vitro for studying numerous functional and morphological alterations in aging brain. Manipulations with the cell expression profiles and activity of senescence-associated molecular machinery within NVU/BBB would allow designing and development of $\mathrm{BBB}$ in vitro models resembling many of aging-coupled phenomena. Table 1 summarizes up-todate data on main molecular mechanisms and markers of aging-associated alterations in NVU cells that are critical for $\mathrm{BBB}$ in vitro modeling and controlling the $\mathrm{BBB}$ integrity.

\section{CONCLUSION AND FUTURE PROSPECTS}

As it is clearly seen from above data, the following approaches could be applied in order to induce senescent phenotype in $\mathrm{BBB}$ cells in vitro: (1) induction of SASP and inflammasome activation; (2) induction of IR; (3) manipulating $\mathrm{NAD}^{+}$levels; (4) induction of DDR associated with $\gamma$-H2AX accumulation; and (5) promotion of mitochondrial biogenesis, glycolytic changes and aberrant production of lactate.

It is clear that application of "standard" $\mathrm{BBB}$ in vitro models to study barrier alterations in the aging brain could be possible if cellular components of the BBB are obtained from the brain of aged animals or animal strains with accelerated aging, i.e., possessing genetically encoded impairments in the signaling of growth hormone/IGF-1, mTOR, sirtuins, suffering from weak antioxidant defense, prone to metabolic alterations, demonstrating prominent inflammatory response or alterations

\section{REFERENCES}

Abbott, N. J., and Friedman, A. (2012). Overview and introduction: the bloodbrain barrier in health and disease. Epilepsia 53, 1-6. doi: 10.1111/j.1528-1167. 2012.03696.x

Akintola, A. A., and van Heemst, D. (2015). Insulin, aging and the brain: mechanisms and implications. Front. Endocrinol. 6:13. doi: 10.3389/fendo. 2015.00013

Akther, S., Korshnova, N., Zhong, J., Liang, M., Cherepanov, S. M., Lopatina, O., et al. (2013). CD38 in the nucleus accumbens and oxytocin are related to paternal behavior in mice. Mol. Brain 6, 41-41. doi: 10.1186/1756-6606-6-41

Albrecht, D. S., Granziera, C., Hooker, J. M., and Loggia, M. L. (2016). In vivo imaging of human neuroinflammation. ACS Chem. Neurosci. 7, 470-483. doi: 10.1021/acschemneuro.6b00056

Antonenko, D., and Flöel, A. (2014). Healthy aging by staying selectively connected: a mini-review. Gerontology 60, 3-9. doi: 10.1159/000354376

Aravinthan, A., Challis, B., Shannon, N., Hoare, M., Heaney, J., and Alexander, G. J. (2015). Selective insulin resistance in hepatocyte senescence. Exp. Cell Res. 331, 38-45. doi: 10.1016/j.yexcr.2014.09.025

Avogaro, A., de Kreutzenberg, S. V., Federici, M., and Fadini, G. P. (2013). The endothelium abridges insulin resistance to premature aging. J. Am. Heart Assoc. 2:e000262. doi: 10.1161/JAHA.113.000262

Bajaj, S., Alkozei, A., Dailey, N. S., and Killgore, W. D. S. (2017). Brain aging: uncovering cortical characteristics of healthy aging in young adults. Front. Aging Neurosci. 9:412. doi: 10.3389/fnagi.2017.00412

Banks, W. A. (2016). From blood-brain barrier to blood-brain interface: new opportunities for CNS drug delivery. Nat. Rev. Drug Discov. 15, 275-292. doi: $10.1038 / \mathrm{nrd} .2015 .21$

Baranowska-Bik, A., and Bik, W. (2017). Insulin and brain aging. Prz. Menopauzalny 16, 44-46. doi: 10.5114/pm.2017.68590 in DNA repair mechanisms, as excellently reviewed in Liao and Kennedy (2014). However, establishment of BBB models using cells isolated from aged animals is usually connected with several technological problems (low rate of cell proliferation, high levels of spontaneous cell death etc.). So, in some cases induction of senescence phenotype should be done in non-aged cells already introduced into the in vitro BBB model (or just prior the reconstitution of the desired multi-cellular ensemble). Therefore, careful phenotyping of cells is absolutely needed for getting correct data on BBB/NVU impairments in the aging brain. Development and application of adequate "aging" $\mathrm{BBB}$ in vitro models would provide further progress in the exploration of brain aging phenomenon, development of novel drug candidates, personification of preventive and therapeutic strategies in patients with age-dependent brain disorders.

\section{AUTHOR CONTRIBUTIONS}

EO conceived and wrote the manuscript. YK performed the literature review and wrote the manuscript. AM, OL, YP, RO and EV performed the literature review and figures design. VS conceived and edited the manuscript. AS conceived, wrote and edited the manuscript.

\section{FUNDING}

This work was supported by the grant given by the President of Russian Federation for the Leading Scientific Teams (Ministry of Education and Science of the Russian Federation; N 6240.2018.7).

Barral, S., Beltramo, R., Salio, C., Aimar, P., Lossi, L., and Merighi, A. (2014). Phosphorylation of histone H2AX in the mouse brain from development to senescence. Int. J. Mol. Sci. 15, 1554-1573. doi: 10.3390/ijms1 5011554

Bauernfeind, F., Niepmann, S., Knolle, P. A., and Hornung, V. (2016). Aging-associated TNF production primes inflammasome activation and NLRP3-related metabolic disturbances. J. Immunol. 197, 2900-2908. doi: 10.4049/jimmunol.1501336

Bell, R. D., Winkler, E. A., Sagare, A. P., Singh, I., LaRue, B., Deane, R., et al. (2010). Pericytes control key neurovascular functions and neuronal phenotype in the adult brain and during brain aging. Neuron 68, 409-427. doi: 10.1016/j.neuron. 2010.09.043

Bernadotte, A., Mikhelson, V. M., and Spivak, I. M. (2016). Markers of cellular senescence. Telomere shortening as a marker of cellular senescence. Aging 8, 3-11. doi: 10.18632/aging.100871

Berthiaume, A.-A., Grant, R. I., McDowell, K. P., Underly, R. G., Hartmann, D. A., Levy, M., et al. (2018). Dynamic remodeling of pericytes in vivo maintains capillary coverage in the adult mouse brain. Cell Rep. 22, 8-16. doi: 10.1016/j. celrep.2017.12.016

Biron, K. E., Dickstein, D. L., Gopaul, R., and Jefferies, W. A. (2011). Amyloid triggers extensive cerebral angiogenesis causing blood brain barrier permeability and hypervascularity in Alzheimer's disease. PLoS One 6:e23789. doi: 10.1371/journal.pone.0023789

Bitto, A., Sell, C., Crowe, E., Lorenzini, A., Malaguti, M., Hrelia, S., et al. (2010). Stress-induced senescence in human and rodent astrocytes. Exp. Cell Res. 316 2961-2968. doi: 10.1016/j.yexcr.2010.06.021

Blau, C. W., Cowley, T. R., O’Sullivan, J., Grehan, B., Browne, T. C., Kelly, L., et al. (2012). The age-related deficit in LTP is associated with changes in perfusion and blood-brain barrier permeability. Neurobiol. Aging 33, 1005.e1023-1005.e1035. doi: 10.1016/j.neurobiolaging.2011.09.035 
Boisvert, M. M., Erikson, G. A., Shokhirev, M. N., and Allen, N. J. (2018). The aging astrocyte transcriptome from multiple regions of the mouse brain. Cell Rep. 22, 269-285. doi: 10.1016/j.celrep.2017.12.039

Boldrini, M., Fulmore, C. A., Tartt, A. N., Simeon, L. R., Pavlova, I., Poposka, V., et al. (2018). Human hippocampal neurogenesis persists throughout aging. Cell Stem Cell 22, 589.e5-599.e5. doi: 10.1016/j.stem.2018.03.015

Bolwerk, C., Govers, L., Knol, H., Oostendorp, T. F., and Brock, R. (2018). Modeling the accumulation of degradable polymer drug carriers in the brain. ChemMedChem 13, 1308-1310. doi: 10.1002/cmdc.201800186

Bonakdar, M., Graybill, P. M., and Davalos, R. V. (2017). A microfluidic model of the blood-brain barrier to study permeabilization by pulsed electric fields. RSC Adv. 7, 42811-42818. doi: 10.1039/c7ra07603g

Borradaile, N. M., and Pickering, J. G. (2009). Nicotinamide phosphoribosyltransferase imparts human endothelial cells with extended replicative lifespan and enhanced angiogenic capacity in a high glucose environment. Aging Cell 8, 100-112. doi: 10.1111/j.1474-9726.2009.00453.x

Bosworth, A. M., Faley, S. L., Bellan, L. M., and Lippmann, E. S. (2018). Modeling neurovascular disorders and therapeutic outcomes with human-induced pluripotent stem cells. Front. Bioeng. Biotechnol. 5:87. doi: 10.3389/fbioe.2017. 00087

Boudina, S. (2013). Cardiac aging and insulin resistance: could insulin/insulin-like growth factor (IGF) signaling be used as a therapeutic target? Curr. Pharm. Des. 19, 5684-5694. doi: 10.2174/1381612811319320004

Brown, J. A., Pensabene, V., Markov, D. A., Allwardt, V., Neely, M. D., Shi, M., et al. (2015). Recreating blood-brain barrier physiology and structure on chip: a novel neurovascular microfluidic bioreactor. Biomicrofluidics 9:054124. doi: $10.1063 / 1.4934713$

Buga, A. M., Margaritescu, C., Scholz, C. J., Radu, E., Zelenak, C., and PopaWagner, A. (2014). Transcriptomics of post-stroke angiogenesis in the aged brain. Front. Aging Neurosci. 6:44. doi: 10.3389/fnagi.2014.00044

Caja, S., and Enríquez, J. A. (2017). Mitochondria in endothelial cells: sensors and integrators of environmental cues. Redox Biol. 12, 821-827. doi: 10.1016/j. redox.2017.04.021

Camacho-Pereira, J., Tarragó, M. G., Chini, C. C. S., Nin, V., Escande, C., Warner, G. M., et al. (2016). CD38 dictates age-related NAD decline and mitochondrial dysfunction through an SIRT3-dependent mechanism. Cell Metab. 23, 1127-1139. doi: 10.1016/j.cmet.2016.05.006

Camandola, S., and Mattson, M. P. (2017). Brain metabolism in health, aging, and neurodegeneration. EMBO J. 36, 1474-1492. doi: 10.15252/embj.201695810

Ceni, C., Pochon, N., Brun, V., Muller-Steffner, H., Andrieux, A., Grunwald, D., et al. (2003). CD38-dependent ADP-ribosyl cyclase activity in developing and adult mouse brain. Biochem. J. 370, 175-183. doi: 10.1042/bj20020604

Cerbai, F., Lana, D., Nosi, D., Petkova-Kirova, P., Zecchi, S., Brothers, H. M., et al. (2012). The neuron-astrocyte-microglia triad in normal brain ageing and in a model of neuroinflammation in the rat hippocampus. PLoS One 7:e45250. doi: 10.1371/journal.pone.0045250

Chan-Ling, T., Hughes, S., Baxter, L., Rosinova, E., McGregor, I., Morcos, Y., et al. (2007). Inflammation and breakdown of the blood-retinal barrier during "physiological aging" in the rat retina: a model for CNS aging. Microcirculation 14, 63-76. doi: 10.1080/10739680601073451

Chavakis, T., Orlova, V. V., and Langer, H. F. (2009). A possible crosstalk between DNA repair pathways and angiogenesis. Cell Cycle 8, 3438-3439. doi: 10.4161/cc.8.21.9750

Chen, J., Brodsky, S. V., Goligorsky, D. M., Hampel, D. J., Li, H., Gross, S. S., et al. (2002). Glycated collagen I induces premature senescence-like phenotypic changes in endothelial cells. Circ. Res. 90, 1290-1298. doi: 10.1161/01.res. 0000022161.42655 .98

Chini, E. N. (2009). CD38 as a regulator of cellular NAD: a novel potential pharmacological target for metabolic conditions. Curr. Pharm. Des. 15, 57-63. doi: $10.2174 / 138161209787185788$

Chini, E. N., Chini, C. C. S., Espindola Netto, J. M., de Oliveira, G. C., and van Schooten, W. (2018). The pharmacology of CD38/NADase: an emerging target in cancer and diseases of aging. Trends Pharmacol. Sci. 39, 424-436. doi: 10.1016/j.tips.2018.02.001

Chinta, S. J., Lieu, C. A., DeMaria, M., Laberge, R.-M., Campisi, J., and Andersen, J. K. (2013). Environmental stress, ageing and glial cell senescence: a novel mechanistic link to Parkinson's disease? J. Inter. Med. 273, 429-436. doi: $10.1111 /$ joim. 12029
Chinta, S. J., Woods, G., Rane, A., Demaria, M., Campisi, J., and Andersen, J. K. (2015). Cellular senescence and the aging brain. Exp. Gerontol. 68, 3-7. doi: 10.1016/j.exger.2014.09.018

Cho, H., Seo, J. H., Wong, K. H. K., Terasaki, Y., Park, J., Bong, K., et al. (2015). Three-dimensional blood-brain barrier model for in vitro studies of neurovascular pathology. Sci. Rep. 5:15222. doi: 10.1038/srep15222

Choi, S. S., Lee, H. J., Lim, I., Satoh, J.-I., and Kim, S. U. (2014). Human astrocytes: secretome profiles of cytokines and chemokines. PLoS One 9:e92325. doi: 10.1371/journal.pone.0092325

Clarke, L. E., Liddelow, S. A., Chakraborty, C., Münch, A. E., Heiman, M., and Barres, B. A. (2018). Normal aging induces A1-like astrocyte reactivity. Proc. Natl. Acad. Sci. U S A 115, E1896-E1905. doi: 10.1073/pnas.1800165115

Cohen, J., D’Agostino, L., Wilson, J., Tuzer, F., and Torres, C. (2017). Astrocyte senescence and metabolic changes in response to HIV antiretroviral therapy drugs. Front. Aging Neurosci. 9:281. doi: 10.3389/fnagi.2017.00281

Coppé, J.-P., Desprez, P.-Y., Krtolica, A., and Campisi, J. (2010). The senescenceassociated secretory phenotype: the dark side of tumor suppression. Annu. Rev. Pathol. 5, 99-118. doi: 10.1146/annurev-pathol-121808-102144

Correia-Melo, C., Marques, F. D., Anderson, R., Hewitt, G., Hewitt, R., Cole, J., et al. (2016). Mitochondria are required for pro-ageing features of the senescent phenotype. EMBO J. 35, 724-742. doi: 10.15252/embj.201592862

Cotelli, M., Manenti, R., Brambilla, M., Zanetti, O., and Miniussi, C. (2012). Naming ability changes in physiological and pathological aging. Front. Neurosci. 6:120. doi: 10.3389/fnins.2012.00120

d'Adda di Fagagna, F. (2008). Living on a break: cellular senescence as a DNA-damage response. Nat. Rev. Cancer 8, 512-522. doi: 10.1038/ nrc2440

da Fonseca, A. C., Matias, D., Garcia, C., Amaral, R., Geraldo, L. H., Freitas, C., et al. (2014). The impact of microglial activation on blood-brain barrier in brain diseases. Front. Cell. Neurosci. 8:362. doi: 10.3389/fncel.2014.00362

De Bock, M., Culot, M., Wang, N., Bol, M., Decrock, E., De Vuyst, E., et al. (2011). Connexin channels provide a target to manipulate brain endothelial calcium dynamics and blood-brain barrier permeability. J. Cereb. Blood Flow Metab. 31, 1942-1957. doi: 10.1038/jcbfm.2011.86

Dejana, E., and Orsenigo, F. (2013). Endothelial adherens junctions at a glance. J. Cell Sci. 126, 2545-2549. doi: 10.1242/jcs. 124529

Dellinger, R. W., Santos, S. R., Morris, M., Evans, M., Alminana, D., Guarente, L., et al. (2017). Repeat dose NRPT (nicotinamide riboside and pterostilbene) increases $\mathrm{NAD}^{+}$levels in humans safely and sustainably: a randomized, double-blind, placebo-controlled study. NPJ Aging Mech. Dis. 3:17. doi: 10.1038/s41514-017-0016-9

Desai, A. K., Grossberg, G. T., and Chibnall, J. T. (2010). Healthy brain aging: a road map. Clin. Geriatr. Med. 26, 1-16. doi: 10.1016/j.cger.2009.12.002

Desai, B. S., Schneider, J. A., Li, J. L., Carvey, P. M., and Hendey, B. (2009). Evidence of angiogenic vessels in Alzheimer's disease. J. Neural Transm. 116, 587-597. doi: 10.1007/s00702-009-0226-9

Desjardins, M., Berti, R., Lefebvre, J., Dubeau, S., and Lesage, F. (2014). Agingrelated differences in cerebral capillary blood flow in anesthetized rats Neurobiol. Aging 35, 1947-1955. doi: 10.1016/j.neurobiolaging.2014.01.136

Donato, A. J., Morgan, R. G., Walker, A. E., and Lesniewski, L. A. (2015). Cellular and molecular biology of aging endothelial cells. J. Mol. Cell. Cardiol. 89, 122-135. doi: 10.1016/j.yjmcc.2015.01.021

Dudvarski Stankovic, N., Teodorczyk, M., Ploen, R., Zipp, F., and Schmidt, M. H. H. (2016). Microglia-blood vessel interactions: a doubleedged sword in brain pathologies. Acta Neuropathol. 131, 347-363. doi: 10.1007/s00401-015-1524-y

Economopoulou, M., Langer, H. F., Celeste, A., Orlova, V. V., Choi, E. Y., Ma, M., et al. (2009). Histone H2AX is integral to hypoxia-driven neovascularisation. Nat. Med. 15, 553-558. doi: 10.1038/nm.1947

Edington, C. D., Chen, W. L. K., Geishecker, E., Kassis, T., Soenksen, L. R., Bhushan, B. M., et al. (2018). Interconnected microphysiological systems for quantitative biology and pharmacology studies. Sci. Rep. 8:4530. doi: 10.1038/s41598-018-22749-0

El Assar, M., Angulo, J., and Rodríguez-Mañas, L. (2013). Oxidative stress and vascular inflammation in aging. Free Radic. Biol. Med. 65, 380-401. doi: 10.1016/j.freeradbiomed.2013.07.003

Elahy, M., Jackaman, C., Mamo, J. C., Lam, V., Dhaliwal, S. S., Giles, C., et al. (2015). Blood-brain barrier dysfunction developed during normal aging is 
associated with inflammation and loss of tight junctions but not with leukocyte recruitment. Immun. Ageing 12:2. doi: 10.1186/s12979-015-0029-9

Enciu, A.-M., Gherghiceanu, M., and Popescu, B. O. (2013). Triggers and effectors of oxidative stress at blood-brain barrier level: relevance for brain ageing and neurodegeneration. Oxid. Med. Cell. Longev. 2013:297512. doi: $10.1155 / 2013 / 297512$

Erdő, F., Denes, L., and de Lange, E. (2017). Age-associated physiological and pathological changes at the blood-brain barrier: a review. J. Cereb. Blood Flow Metab. 37, 4-24. doi: 10.1177/0271678x16679420

Freeman, L. R., and Keller, J. N. (2012). Oxidative stress and cerebral endothelial cells: regulation of the blood-brain-barrier and antioxidant based interventions. Biochim. Biophys. Acta 1822, 822-829. doi: 10.1016/j.bbadis.2011.12.009

Frölich, L., Blum-Degen, D., Bernstein, H. G., Engelsberger, S., Humrich, J., Laufer, S., et al. (1998). Brain insulin and insulin receptors in aging and sporadic Alzheimer's disease. J. Neural Transm. 105, 423-438. doi: $10.1007 /$ s007020050068

Fu, H. Q., Yang, T., Xiao, W., Fan, L., Wu, Y., Terrando, N., et al. (2014). Prolonged neuroinflammation after lipopolysaccharide exposure in aged rats. PLoS One 9:e106331. doi: 10.1371/journal.pone.0106331

Garberg, P., Ball, M., Borg, N., Cecchelli, R., Fenart, L., Hurst, R. D., et al. (2005). In vitro models for the blood-brain barrier. Toxicol. in Vitro 19, 299-334. doi: 10.1016/j.tiv.2004.06.011

Ghosh, K., Agarwal, P., and Haggerty, G. (2011). Alzheimer's disease-not an exaggeration of healthy aging. Indian J. Psychol. Med. 33, 106-114. doi: $10.4103 / 0253-7176.92047$

Goligorsky, M. S., and Hirschi, K. (2016). Stress-induced premature senescence of endothelial and endothelial progenitor cells. Adv. Pharmacol. 77, 281-306. doi: 10.1016/bs.apha.2016.04.007

Goodall, E. F., Wang, C., Simpson, J. E., Baker, D. J., Drew, D. R., Heath, P. R., et al. (2018). Age-associated changes in the blood-brain barrier: comparative studies in human and mouse. Neuropathol. Appl. Neurobiol. 44, 328-340. doi: $10.1111 /$ nan. 12408

Greenberg, D. L., Fetzer, D. L., Payne, M. E., MacFall, J. R., Provenzale, J. M., Steffens, D. C., et al. (2008). Aging, gender, and the elderly adult brain: an examination of analytical strategies. Neurobiol. Aging 29, 290-302. doi: 10.1016/j.neurobiolaging.2006.09.016

Guerreiro, R., and Bras, J. (2015). The age factor in Alzheimer's disease. Genome Med. 7:106. doi: 10.1186/s13073-015-0232-5

Helms, H. C., Abbott, N. J., Burek, M., Cecchelli, R., Couraud, P. O., Deli, M. A., et al. (2016). In vitro models of the blood-brain barrier: an overview of commonly used brain endothelial cell culture models and guidelines for their use. J. Cereb. Blood Flow Metab. 36, 862-890. doi: 10.1177/0271678x16 630991

Hicks, P., Rolsten, C., Brizzee, D., and Samorajski, T. (1983). Age-related changes in rat brain capillaries. Neurobiol. Aging 4, 69-75. doi: 10.1016/01974580(83)90057-x

Higashida, H., Yokoyama, S., Kikuchi, M., and Munesue, T. (2012). CD38 and its role in oxytocin secretion and social behavior. Horm. Behav. 61, 351-358. doi: 10.1016/j.yhbeh.2011.12.011

Hoffmann, J., Haendeler, J., Aicher, A., Rössig, L., Vasa, M., Zeiher, A. M., et al. (2001). Aging enhances the sensitivity of endothelial cells toward apoptotic stimuli: important role of nitric oxide. Circ. Res. 89, 709-715. doi: 10.1161/hh2001.097796

Hughes, S., Gardiner, T., Hu, P., Baxter, L., Rosinova, E., and Chan-Ling, T. (2006). Altered pericyte-endothelial relations in the rat retina during aging: implications for vessel stability. Neurobiol. Aging 27, 1838-1847. doi: 10.1016/j. neurobiolaging.2005.10.021

James, E. L., Michalek, R. D., Pitiyage, G. N., de Castro, A. M., Vignola, K. S., Jones, J., et al. (2015). Senescent human fibroblasts show increased glycolysis and redox homeostasis with extracellular metabolomes that overlap with those of irreparable DNA damage, aging and disease. J. Proteome Res. 14, 1854-1871. doi: $10.1021 /$ pr501221g

Jiang, T., and Cadenas, E. (2014). Astrocytic metabolic and inflammatory changes as a function of age. Aging Cell 13, 1059-1067. doi: 10.1111/acel. 12268

Karnewar, S., Neeli, P. K., Panuganti, D., Kotagiri, S., Mallappa, S., Jain, N., et al. (2018). Metformin regulates mitochondrial biogenesis and senescence through AMPK mediated H3K79 methylation: relevance in age-associated vascular dysfunction. Biochim. Biophys. Acta 1864, 1115-1128. doi: 10.1016/j.bbadis. 2018.01.018

Katsimpardi, L., Litterman, N. K., Schein, P. A., Miller, C. M., Loffredo, F. S., Wojtkiewicz, G. R., et al. (2014). Vascular and neurogenic rejuvenation of the aging mouse brain by young systemic factors. Science 344, 630-634. doi: 10.1126/science.1251141

Khilazheva, E. D., Boytsova, E. B., Pozhilenkova, E. A., Solonchuk, Y. R., and Salmina, A. B. (2015). The model of neurovascular unit in vitro consisting of three cells types. Tsitologiia 57, 710-713.

Khilazheva, E. D., Pisareva, N. V., Morgun, A. V., Boytsova, E. B., Taranushenko, T. E., Frolova, O. V., et al. (2017). Activation of GPR81 lactate receptors stimulates mitochondrial biogenesis in cerebral microvessel endothelial cells. Ann. Clin. Exp. Neurol. 11, 34-39.

Kiraly, A., Szabo, N., Toth, E., Csete, G., Farago, P., Kocsis, K., et al. (2016). Male brain ages faster: the age and gender dependence of subcortical volumes. Brain Imaging Behav. 10, 901-910. doi: 10.1007/s11682-015-9468-3

Kluge, M. A., Fetterman, J. L., and Vita, J. A. (2013). Mitochondria and endothelial function. Circ. Res. 112, 1171-1188. doi: 10.1161/CIRCRESAHA.111.300233

Kress, B. T., Iliff, J. J., Xia, M., Wang, M., Wei, H. S., Zeppenfeld, D., et al. (2014). Impairment of paravascular clearance pathways in the aging brain. Ann. Neurol. 76, 845-861. doi: 10.1002/ana.24271

Kuvacheva, N. V., Morgun, A., Khilazheva, E., Boytsova, E. B., Ruzaeva, V. A., Shuvaev, A., et al. (2016). Blood brain barrier cell proliferation under HIF-1 suppressed activity in vitro. Sib. Med. Rev. 98, 51-56. doi: 10.20333/250001362016-2-51-56

LaBarbera, K. E., Hyldahl, R. D., O'Fallon, K. S., Clarkson, P. M., and Witkowski, S. (2015). Pericyte NF-кB activation enhances endothelial cell proliferation and proangiogenic cytokine secretion in vitro. Physiol. Rep. 3:e12309. doi: 10.14814/phy2.12309

Lecuyer, M. A., Kebir, H., and Prat, A. (2016). Glial influences on BBB functions and molecular players in immune cell trafficking. Biochim. Biophys. Acta 1862, 472-482. doi: 10.1016/j.bbadis.2015.10.004

Lee, P., Kim, J., Williams, R., Sandhir, R., Gregory, E., Brooks, W. M., et al. (2012). Effects of aging on blood brain barrier and matrix metalloproteases following controlled cortical impact in mice. Exp. Neurol. 234, 50-61. doi: 10.1016/j. expneurol.2011.12.016

Lei, M., Zhu, Z., Wen, Z., and Ke, S. (2013). Impairments of tight junctions are involved in D-galactose-induced brain aging. Neuroreport 24, 671-676. doi: 10.1097/WNR.0b013e3283638f75

Li, F., Lan, Y., Wang, Y., Wang, J., Yang, G., Meng, F., et al. (2011). Endothelial Smad4 maintains cerebrovascular integrity by activating $\mathrm{N}$-cadherin through cooperation with notch. Dev. Cell 20, 291-302. doi: 10.1016/j.devcel.2011. 01.011

Li, Y., Smith, D., Li, Q., Sheibani, N., Huang, S., Kern, T., et al. (2012). Antibodymediated retinal pericyte injury: implications for diabetic retinopathy. Invest. Ophthal. Vis. Sci. 53, 5520-5526. doi: 10.1167/iovs.12-10010

Liao, C. Y., and Kennedy, B. K. (2014). Mouse models and aging: longevity and progeria. Curr. Top. Dev. Biol. 109, 249-285. doi: 10.1016/B978-0-12-3979209.00003-2

Liebner, S., Corada, M., Bangsow, T., Babbage, J., Taddei, A., Czupalla, C. J., et al. (2008). Wnt/ $\beta$-catenin signaling controls development of the blood-brain barrier. J. Cell Biol. 183, 409-417. doi: 10.1083/jcb.200806024

Lin, R., Cai, J., Nathan, C., Wei, X., Schleidt, S., Rosenwasser, R., et al. (2015). Neurogenesis is enhanced by stroke in multiple new stem cell niches along the ventricular system at sites of high BBB permeability. Neurobiol. Dis. 74, 229-239. doi: 10.1016/j.nbd.2014.11.016

Lippmann, E. S., Weidenfeller, C., Svendsen, C. N., and Shusta, E. V. (2011). Blood-brain barrier modeling with co-cultured neural progenitor cell-derived astrocytes and neurons. J. Neurochem. 119, 507-520. doi: 10.1111/j.1471-4159. 2011.07434.x

Lopatina, O., Inzhutova, A., Pichugina, Y. A., Okamoto, H., Salmina, A. B., and Higashida, H. (2011). Reproductive experience affects parental retrieval behaviour associated with increased plasma oxytocin levels in wild-type and CD38-knockout mice. J. Neuroendocrinol. 23, 1125-1133. doi: 10.1111/j.13652826.2011.02136.x

Lopatina, O., Inzhutova, A., Salmina, A. B., and Higashida, H. (2012). The roles of oxytocin and CD38 in social or parental behaviors. Front. Neurosci. 6:182. doi: $10.3389 /$ fnins.2012.00182 
Luissint, A.-C., Artus, C., Glacial, F., Ganeshamoorthy, K., and Couraud, P.-O. (2012). Tight junctions at the blood brain barrier: physiological architecture and disease-associated dysregulation. Fluids Barriers CNS 9, 23-23. doi: 10.1186/2045-8118-9-23

Madabhushi, R., Gao, F., Pfenning, A. R., Pan, L., Yamakawa, S., Seo, J., et al. (2015). Activity-induced DNA breaks govern the expression of neuronal earlyresponse genes. Cell 161, 1592-1605. doi: 10.1016/j.cell.2015.05.032

Maicher, A., Kastner, L., Dees, M., and Luke, B. (2012). Deregulated telomere transcription causes replication-dependent telomere shortening and promotes cellular senescence. Nucleic Acids Res. 40, 6649-6659. doi: 10.1093/nar/ gks358

Malavasi, F., Deaglio, S., Ferrero, E., Funaro, A., Sancho, J., Ausiello, C. M., et al. (2006). CD38 and CD157 as receptors of the immune system: a bridge between innate and adaptive immunity. Mol. Med. 12, 334-341. doi: 10.2119/200600094.Molmed

Malavasi, F., Deaglio, S., Funaro, A., Ferrero, E., Horenstein, A. L., Ortolan, E., et al. (2008). Evolution and function of the ADP ribosyl cyclase/CD38 gene family in physiology and pathology. Physiol. Rev. 88, 841-886. doi: 10.1152/physrev.00035.2007

Małecki, A., Skipor-Lahuta, J., Toborek, M., Abbott, N. J., Antonetti, D. A., Su, E. J., et al. (2017). Abstracts from the 20th international symposium on signal transduction at the blood-brain barriers. Fluids Barriers CNS 14:27. doi: 10.1186/s12987-017-0071-4

Malinovskaya, N. A., Komleva, Y. K., Salmin, V. V., Morgun, A. V., Shuvaev, A. N., Panina, Y. A., et al. (2016). Endothelial progenitor cells physiology and metabolic plasticity in brain angiogenesis and blood-brain barrier modeling. Front. Physiol. 7:599. doi: 10.3389/fphys.2016.00599

Marques, F., Sousa, J. C., Sousa, N., and Palha, J. A. (2013). Blood-brainbarriers in aging and in Alzheimer's disease. Mol. Neurodegener. 8, 38-38. doi: 10.1186/1750-1326-8-38

Montagne, A., Barnes, S. R., Sweeney, M. D., Halliday, M. R., Sagare, A. P., Zhao, Z., et al. (2015). Blood-brain barrier breakdown in the aging human hippocampus. Neuron 85, 296-302. doi: 10.1016/j.neuron.2014.12.032

Mooradian, A. D., Morin, A. M., Cipp, L. J., and Haspel, H. C. (1991). Glucose transport is reduced in the blood-brain barrier of aged rats. Brain Res. 551, 145-149. doi: 10.1016/0006-8993(91)90926-m

Morgan, R. G., Donato, A. J., and Walker, A. E. (2018). Telomere uncapping and vascular aging. Am. J. Physiol. Heart Circ. Physiol. 315, H1-H5. doi: 10.1152/ajpheart.00008.2018

Morrison, J. H., and Baxter, M. G. (2012). The aging cortical synapse: hallmarks and implications for cognitive decline. Nat. Rev. Neurosci. 13, 240-250. doi: $10.1038 / \mathrm{nrn} 3200$

Mortuza, R., Chen, S., Feng, B., Sen, S., and Chakrabarti, S. (2013). High glucose induced alteration of SIRTs in endothelial cells causes rapid aging in a p300 and FOXO regulated pathway. PLoS One 8:e54514. doi: 10.1371/journal.pone. 0054514

Mosconi, L. (2013). Glucose metabolism in normal aging and Alzheimer's disease: Methodological and physiological considerations for PET studies. Clin. Transl. Imaging 1, 217-233. doi: 10.1007/s40336-013-0026-y

Mostany, R., Anstey, J. E., Crump, K. L., Maco, B., Knott, G., and PorteraCailliau, C. (2013). Altered synaptic dynamics during normal brain aging. J. Neurosci. 33, 4094-4104. doi: 10.1523/JNEUROSCI.4825-12.2013

Muche, A., Arendt, T., and Schliebs, R. (2017). Oxidative stress affects processing of amyloid precursor protein in vascular endothelial cells. PLoS One 12:e0178127. doi: 10.1371/journal.pone.0178127

Mullins, R. J., Diehl, T. C., Chia, C. W., and Kapogiannis, D. (2017). Insulin resistance as a link between amyloid- $\beta$ and tau pathologies in Alzheimer's disease. Front. Aging Neurosci. 9:118. doi: 10.3389/fnagi.2017.00118

Myung, N.-H., Zhu, X., Kruman, I. I., Castellani, R. J., Petersen, R. B., Siedlak, S. L., et al. (2008). Evidence of DNA damage in Alzheimer disease: phosphorylation of histone H2AX in astrocytes. Age 30, 209-215. doi: 10.1007/s11357-0089050-7

Neth, B. J., and Craft, S. (2017). Insulin resistance and Alzheimer's disease: bioenergetic linkages. Front. Aging Neurosci. 9:345. doi: 10.3389/fnagi.2017. 00345

Norden, D. M., and Godbout, J. P. (2013). Microglia of the aged brain: primed to be activated and resistant to regulation. Neuropathol. Appl. Neurobiol. 39, 19-34. doi: 10.1111/j.1365-2990.2012.01306.x
Oakley, R., and Tharakan, B. (2014). Vascular hyperpermeability and aging. Aging Dis. 5, 114-125. doi: 10.14336/AD.2014.0500114

Osipova, E. D., Semyachkina-Glushkovskaya, O. V., Morgun, A. V., Pisareva, N. V., Malinovskaya, N. A., Boitsova, E. B., et al. (2018). Gliotransmitters and cytokines in the control of blood-brain barrier permeability. Rev. Neurosci. 29, 567-591. doi: 10.1515/revneuro-2017 $-0092$

Pacher, P., Mabley, J. G., Soriano, F. G., Liaudet, L., Komjati, K., and Szabo, C. (2002). Endothelial dysfunction in aging animals: the role of poly(ADP-ribose) polymerase activation. Br. J. Pharmacol. 135, 1347-1350. doi: 10.1038/sj.bjp. 0704627

Peters, R. (2006). Ageing and the brain. Postgrad. Med. J. 82, 84-88. doi: 10.1136/pgmj.2005.036665

Phan, D. T., Bender, R. H. F., Andrejecsk, J. W., Sobrino, A., Hachey, S. J., George, S. C., et al. (2017). Blood-brain barrier-on-a-chip: microphysiological systems that capture the complexity of the blood-central nervous system interface. Exp. Biol. Med. 242, 1669-1678. doi: 10.1177/1535370217694100

Piantadosi, C. A., and Suliman, H. B. (2012). Transcriptional control of mitochondrial biogenesis and its interface with inflammatory processes. Biochim. Biophys. Acta 1820, 532-541. doi: 10.1016/j.bbagen.2012.01.003

Pozhilenkova, E. A., Lopatina, O. L., Komleva, Y. K., Salmin, V. V., and Salmina, A. B. (2017). Blood-brain barrier-supported neurogenesis in healthy and diseased brain. Rev. Neurosci. 28, 397-415. doi: 10.1515/revneuro2016-0071

Rao, R. (2008). Oxidative stress-induced disruption of epithelial and endothelial tight junctions. Front. Biosci. 13, 7210-7226. doi: 10.2741/3223

Reyes, L. A., Boslett, J., Varadharaj, S., De Pascali, F., Hemann, C., Druhan, L. J., et al. (2015). Depletion of $\operatorname{NADP}(H)$ due to $\mathrm{CD} 38$ activation triggers endothelial dysfunction in the postischemic heart. Proc. Natl. Acad. Sci. U S A 112, 11648-11653. doi: 10.1073/pnas. 1505556112

Ribecco-Lutkiewicz, M., Sodja, C., Haukenfrers, J., Haqqani, A. S., Ly, D., Zachar, P., et al. (2018). A novel human induced pluripotent stem cell blood-brain barrier model: applicability to study antibody-triggered receptor-mediated transcytosis. Sci. Rep. 8:1873. doi: 10.1038/s41598-018-1 9522-8

Ritschka, B., Storer, M., Mas, A., Heinzmann, F., Ortells, M. C., Morton, J. P., et al. (2017). The senescence-associated secretory phenotype induces cellular plasticity and tissue regeneration. Genes Dev. 31, 172-183. doi: 10.1101/gad. 290635.116

Robbins, P. D. (2017). Extracellular vesicles and aging. Stem Cell Investig. 4:98. doi: $10.21037 /$ sci.2017.12.03

Rodríguez, J. J., Yeh, C.-Y., Terzieva, S., Olabarria, M., Kulijewicz-Nawrot, M., and Verkhratsky, A. (2014). Complex and region-specific changes in astroglial markers in the aging brain. Neurobiol. Aging 35, 15-23. doi: 10.1016/j. neurobiolaging.2013.07.002

Ross, J. M., Öberg, J., Brené, S., Coppotelli, G., Terzioglu, M., Pernold, K., et al. (2010). High brain lactate is a hallmark of aging and caused by a shift in the lactate dehydrogenase A/B ratio. Proc. Natl. Acad. Sci. U S A 107, 20087-20092. doi: 10.1073/pnas.1008189107

Ruck, T., Bittner, S., and Meuth, S. G. (2015). Blood-brain barrier modeling: challenges and perspectives. Neural Regen. Res. 10, 889-891. doi: 10.4103/16735374.158342

Salmin, V. V., Komleva, Y. K., Kuvacheva, N. V., Morgun, A. V., Khilazheva, E. D., Lopatina, O. L., et al. (2017). Differential roles of environmental enrichment in Alzheimer's type of neurodegeneration and physiological aging. Front. Aging Neurosci. 9:245. doi: 10.3389/fnagi.2017.00245

Salmina, A. B., Inzhutova, A. I., Morgun, A. V., Okuneva, O. S., Malinovskaia, N. A., Lopatina, O. L., et al. (2012). NAD ${ }^{+}$-converting enzymes in neuronal and glial cells: CD38 as a novel target for neuroprotection. Vestn. Akad. Med. Nauk 10, 29-37.

Salmina, A. B., Komleva, Y. K., Szijártó, I. A., Gorina, Y. V., Lopatina, O. L., Gertsog, G. E., et al. (2015a). $\mathrm{H}_{2} \mathrm{~S}$ - and NO-signaling pathways in Alzheimer's amyloid vasculopathy: synergism or antagonism? Front. Physiol. 6:361. doi: 10.3389/fphys.2015.00361

Salmina, A. B., Kuvacheva, N. V., Morgun, A. V., Komleva, Y. K., Pozhilenkova, E. A., Lopatina, O. L., et al. (2015b). Glycolysis-mediated control of blood-brain barrier development and function. Int. J. Biochem. Cell Biol. 64, 174-184. doi: 10.1016/j.biocel.2015.04.005 
Semyachkina-Glushkovskaya, O., Abdurashitov, A., Dubrovsky, A., Bragin, D., Bragina, O., Shushunova, N., et al. (2017a). Application of optical coherence tomography for in vivo monitoring of the meningeal lymphatic vessels during opening of blood-brain barrier: mechanisms of brain clearing. J. Biomed. Opt. 22, 1-9. doi: 10.1117/1.JBO.22.12.121719

Semyachkina-Glushkovskaya, O., Kurths, J., Borisova, E., Sokolovski, S., Mantareva, V., Angelov, I., et al. (2017b). Photodynamic opening of bloodbrain barrier. Biomed. Opt. Express 8, 5040-5048. doi: 10.1364/BOE.8. 005040

Seo, J., Kim, S. C., Lee, H.-S., Kim, J. K., Shon, H. J., Salleh, N. L. M., et al. (2012). Genome-wide profiles of $\mathrm{H} 2 \mathrm{AX}$ and $\gamma-\mathrm{H} 2 \mathrm{AX}$ differentiate endogenous and exogenous DNA damage hotspots in human cells. Nucleic Acids Res. 40, 5965-5974. doi: 10.1093/nar/gks287

Silverberg, G. D., Messier, A. A., Miller, M. C., Machan, J. T., Majmudar, S. S., Stopa, E. G., et al. (2010). Amyloid efflux transporter expression at the blood-brain barrier declines in normal aging. J. Neuropathol. Exp. Neurol. 69, 1034-1043. doi: 10.1097/NEN.0b013e3181f46e25

Sohrabji, F., Bake, S., and Lewis, D. K. (2013). Age-related changes in brain support cells: implications for stroke severity. Neurochem. Int. 63, 291-301. doi: 10.1016/j.neuint.2013.06.013

Solano Fonseca, R., Mahesula, S., Apple, D. M., Raghunathan, R., Dugan, A., Cardona, A., et al. (2016). Neurogenic niche microglia undergo positional remodeling and progressive activation contributing to age-associated reductions in neurogenesis. Stem Cells Dev. 25, 542-555. doi: 10.1089/scd. 2015.0319

Spittau, B. (2017). Aging microglia-phenotypes, functions and implications for age-related neurodegenerative diseases. Front. Aging Neurosci. 9:194. doi: 10.3389/fnagi.2017.00194

Stanimirovic, D. B., Bani-Yaghoub, M., Perkins, M., and Haqqani, A. S. (2015). Blood-brain barrier models: in vitro to in vivo translation in preclinical development of CNS-targeting biotherapeutics. Expert Opin. Drug Discov. 10, 141-155. doi: 10.1517/17460441.2015.974545

Suberbielle, E., Sanchez, P. E., Kravitz, A. V., Wang, X., Ho, K., Eilertson, K., et al. (2013). Physiological brain activity causes DNA double-strand breaks in neurons, with exacerbation by amyloid- $\beta$. Nat. Neurosci. 16, 613-621. doi: 10.1038/nn.3356

Sun, D., McGinn, M., Hankins, J. E., Mays, K. M., Rolfe, A., and Colello, R. J. (2013). Aging- and injury-related differential apoptotic response in the dentate gyrus of the hippocampus in rats following brain trauma. Front. Aging Neurosci. 5:95. doi: 10.3389/fnagi.2013.00095

Thambisetty, M., Beason-Held, L. L., An, Y., Kraut, M., Metter, J., Egan, J., et al. (2013). Impaired glucose tolerance in midlife and longitudinal changes in brain function during aging. Neurobiol. Aging 34, 2271-2276. doi: 10.1016/j. neurobiolaging.2013.03.025

Tschopp, J. (2011). Mitochondria: sovereign of inflammation? Eur. J. Immunol. 41, 1196-1202. doi: 10.1002/eji.201141436

Tucsek, Z., Toth, P., Sosnowska, D., Gautam, T., Mitschelen, M., Koller, A., et al. (2014). Obesity in aging exacerbates blood-brain barrier disruption, neuroinflammation, and oxidative stress in the mouse hippocampus: effects on expression of genes involved in $\beta$-amyloid generation and Alzheimer's disease. J. Gerontol. A Biol. Sci. Med. Sci. 69, 1212-1226. doi: 10.1093/gerona/glt177

Turinetto, V., and Giachino, C. (2015). Multiple facets of histone variant H2AX: a DNA double-strand-break marker with several biological functions. Nucleic Acids Res. 43, 2489-2498. doi: 10.1093/nar/gkv061

van Assema, D. M., Lubberink, M., Boellaard, R., Schuit, R. C., Windhorst, A. D., Scheltens, P., et al. (2012). P-glycoprotein function at the blood-brain barrier: effects of age and gender. Mol. Imaging Biol. 14, 771-776. doi: 10.1007/s11307012-0556-0

Villaseñor, R., Kuennecke, B., Ozmen, L., Ammann, M., Kugler, C., Grüninger, F., et al. (2017). Region-specific permeability of the bloodbrain barrier upon pericyte loss. J. Cereb. Blood Flow Metab. 37, 3683-3694. doi: $10.1177 / 0271678 \times 17697340$

Wakabayashi, T., Naito, H., Suehiro, J.-I., Lin, Y., Kawaji, H., Iba, T., et al. (2018). CD157 marks tissue-resident endothelial stem cells with homeostatic and regenerative properties. Cell Stem Cell 22, 384-397. doi: 10.1016/j.stem. 2018.01.010
Watanabe, S., Kawamoto, S., Ohtani, N., and Hara, E. (2017). Impact of senescence-associated secretory phenotype and its potential as a therapeutic target for senescence-associated diseases. Cancer Sci. 108, 563-569. doi: $10.1111 /$ cas. 13184

Willette, A. A., Modanlo, N., and Kapogiannis, D. (2015). Insulin resistance predicts medial temporal hypermetabolism in mild cognitive impairment conversion to Alzheimer disease. Diabetes 64, 1933-1940. doi: 10.2337/db141507

Winkler, E. A., Bell, R. D., and Zlokovic, B. V. (2010). Pericyte-specific expression of PDGF $\beta$ receptor in mouse models with normal and deficient PDGF $\beta$ receptor signaling. Mol. Neurodegenr. 5:32. doi: 10.1186/17501326-5-32

Woolard, J., Bevan, H. S., Harper, S. J., and Bates, D. O. (2009). Molecular diversity of VEGF-A as a regulator of its biological activity. Microcirculation 16, 572-592. doi: $10.1080 / 10739680902997333$

Yamazaki, Y., Baker, D. J., Tachibana, M., Liu, C. C., van Deursen, J. M., Brott, T. G., et al. (2016). Vascular cell senescence contributes to bloodbrain barrier breakdown. Stroke 47, 1068-1077. doi: 10.1161/STROKEAHA. 115.010835

Yamazaki, Y., and Kanekiyo, T. (2017). Blood-brain barrier dysfunction and the pathogenesis of Alzheimer's disease. Int. J. Mol. Sci. 18:E1965. doi: $10.3390 / \mathrm{ijms} 18091965$

Yin, Y., Zhou, Z., Liu, W., Chang, Q., Sun, G., and Dai, Y. (2017). Vascular endothelial cells senescence is associated with NOD-like receptor family pyrin domain-containing 3 (NLRP3) inflammasome activation via reactive oxygen species (ROS)/thioredoxin-interacting protein (TXNIP) pathway. Int. J. Biochem. Cell Biol. 84, 22-34. doi: 10.1016/j.biocel.2017. 01.001

Zeevi, N., Pachter, J., McCullough, L. D., Wolfson, L., and Kuchel, G. A. (2010). The blood-brain barrier: geriatric relevance of a critical brain-body interface. J. Am. Geriatr. Soc. 58, 1749-1757. doi: 10.1111/j.1532-5415.2010. 03011.x

Zhang, X., Fan, Z., and Jin, T. (2017). Crocin protects against cerebralischemia-induced damage in aged rats through maintaining the integrity of blood-brain barrier. Restor. Neurol. Neurosci. 35, 65-75. doi: 10.3233/RNN-1 60696

Zhang, Y. Y., Liu, H., Summerfield, S. G., Luscombe, C. N., and Sahi, J. (2016). Integrating in silico and in vitro approaches to predict drug accessibility to the central nervous system. Mol. Pharm. 13, 1540-1550. doi: 10.1021/acs. molpharmaceut.6b00031

Zhang, H., Ryu, D., Wu, Y., Gariani, K., Wang, X., Luan, P., et al. (2016). NAD ${ }^{+}$ repletion improves mitochondrial and stem cell function and enhances life span in mice. Science 352, 1436-1443. doi: 10.1126/science.aaf2693

Zhao, L., Mao, Z., Woody, S. K., and Brinton, R. D. (2016). Sex differences in metabolic aging of the brain: insights into female susceptibility to Alzheimer's disease. Neurobiol. Aging 42, 69-79. doi: 10.1016/j.neurobiolaging.2016. 02.011

Zhou, Y. F., Li, Y. N., Jin, H. J., Wu, J. H., He, Q. W., Wang, X. X., et al. (2018). Sema4D/PlexinB1 inhibition ameliorates blood-brain barrier damage and improves outcome after stroke in rats. FASEB J. 32, 2181-2196. doi: 10.1096/fj. 201700786RR

Zöller, T., Attaai, A., Potru, P. S., Ruß, T., and Spittau, B. (2018). Aged mouse cortical microglia display an activation profile suggesting immunotolerogenic functions. Int. J. Mol. Sci. 19:E706. doi: 10.3390/ijms19030706

Conflict of Interest Statement: The authors declare that the research was conducted in the absence of any commercial or financial relationships that could be construed as a potential conflict of interest.

Copyright $\odot 2018$ Osipova, Komleva, Morgun, Lopatina, Panina, Olovyannikova, Vais, Salmin and Salmina. This is an open-access article distributed under the terms of the Creative Commons Attribution License (CC BY). The use, distribution or reproduction in other forums is permitted, provided the original author(s) and the copyright owner(s) are credited and that the original publication in this journal is cited, in accordance with accepted academic practice. No use, distribution or reproduction is permitted which does not comply with these terms. 